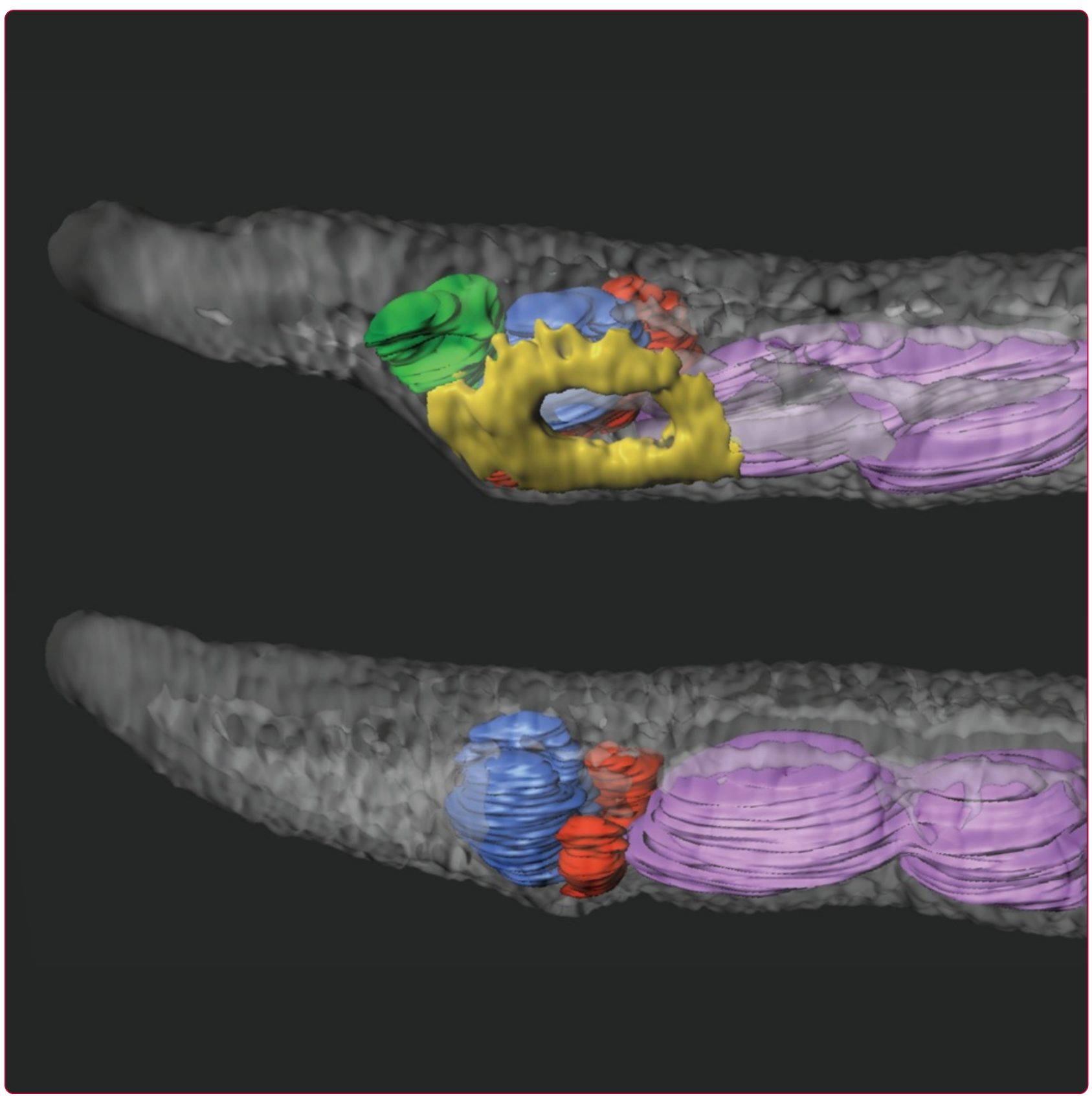

The Nodal signaling pathway controls left-right asymmetric development in amphioxus

Soukup et al.

() Biomed Central 


\title{
The Nodal signaling pathway controls left-right asymmetric development in amphioxus
}

\author{
Vladimir Soukup ${ }^{1}$, Luok Wen Yong ${ }^{2}$, Tsai-Ming Lu², Song-Wei Huang ${ }^{2}$, Zbynek Kozmik ${ }^{*^{*}}$ and Jr-Kai Yu ${ }^{2,3^{*}}$
}

\begin{abstract}
Background: Nodal is an important determinant of the left-right (LR) body axis in bilaterians, specifying the right side in protostomes and non-chordate deuterostomes as opposed to the left side in chordates. Amphioxus represents an early-branching chordate group, rendering it especially useful for studying the character states that predate the origin of vertebrates. However, its anatomy, involving offset arrangement of axial structures, marked asymmetry of the oropharyngeal region, and, most notably, a mouth positioned on the left side, contrasts with the symmetric arrangement of the corresponding regions in other chordates.

Results: We show that the Nodal signaling pathway acts to specify the LR axis in the cephalochordate amphioxus in a similar way as in vertebrates. At early neurula stages, Nodal switches from initial bilateral to the left-sided expression and subsequently specifies the left embryonic side. Perturbation of Nodal signaling with small chemical inhibitors (SB505124 and SB431542) alters expression of other members of the pathway and of left/right-sided, organ-specific genes. Upon inhibition, larvae display loss of the innate alternation of both somites and axons of peripheral nerves and loss of left-sided pharyngeal structures, such as the mouth, the preoral pit, and the duct of the club-shaped gland. Concomitantly, the left side displays ectopic expression of otherwise right-sided genes, and the larvae exhibit bilaterally symmetrical morphology, with duplicated endostyle and club-shaped gland structures.

Conclusions: We demonstrate that Nodal signaling is necessary for establishing the LR embryonic axis and for developing profound asymmetry in amphioxus. Our data suggest that initial symmetry breaking in amphioxus and propagation of the pathway on the left side correspond with the situation in vertebrates. However, the organs that become targets of the pathway differ between amphioxus and vertebrates, which may explain the pronounced asymmetry of its oropharyngeal and axial structures and the left-sided position of the mouth.
\end{abstract}

Keywords: Nodal signaling, Amphioxus, Left-right asymmetry, Mouth opening, Embryonic development

\section{Background}

Bilaterians exhibit varying degrees of asymmetry along the left-right (LR) body axis. Most animals exhibit a symmetrical outward appearance with conserved directional asymmetry of the visceral organs, while others, such as snails and crabs, possess strikingly asymmetrical external features [1-3]. Many embryos usually undergo an initial symmetrybreaking event, which is followed by the asymmetrical propagation of signaling cues and gene expression, and eventually asymmetrical organ formation [4]. Although the

\footnotetext{
* Correspondence: kozmik@img.cas.cz; jkyu@gate.sinica.edu.tw

'Institute of Molecular Genetics, Academy of Sciences of the Czech Republic, Videnska 1083, Prague 14220, Czech Republic

${ }^{2}$ Institute of Cellular and Organismic Biology, Academia Sinica, 128 Academia Road, Section 2, Nankang, Taipei 11529, Taiwan

Full list of author information is available at the end of the article
}

nature of the symmetry-breaking event varies between different animals [5-7], the signaling cascade that patterns the LR axis seems to be highly conserved $[1,2,8]$.

Nodal, a transforming growth factor beta (TGF- $\beta$ ) superfamily factor, has been previously identified to be central to determining LR asymmetry through unilateral activation of downstream genes. In mouse, Nodal is initially expressed bilaterally around the node, where the encoded protein interacts with its co-ligand GDF1. The Nodal/GDF1 heterodimer exhibits higher activity than the Nodal homodimer and also acts at a longer range [9]. The action of the Nodal inhibitor Cerl2 on the right side ensures that Nodal becomes preferentially active on the left side, and this activity is transferred to the left lateral plate mesoderm [10-12]. Here, Nodal activates its own expression and also triggers expression of the TGF- $\beta$ 
factor Lefty2 and transcription factor Pitx2. Lefty2, which diffuses at a high velocity and inhibits Nodal, ensures that Nodal becomes restricted to the left side; here, Nodal further enhances its own expression and expression of Lefty 2 and Pitx2 [13]. Pitx2 subsequently triggers expression of downstream targets and promotes tissue-specific proliferation and differentiation, leading to asymmetrical development of the affected organs ([14] and literature therein).

Nodal signaling has been identified in most deuterostomes, where it determines both internal organ asymmetry and asymmetric external development [14-18]. In protostomes, Nodal signaling directs shell coiling in snails and probably also LR asymmetry of internal organs in other lophotrochozoans; on the other hand, Nodal has not been identified in ecdysozoans, despite numerous examples of directional LR asymmetries in these organisms [19-21]. Interestingly, the recent finding that the Nodal-Pitx cascade is responsible for asymmetric budding and branching morphogenesis of polyps in Hydra suggests that regulation of asymmetric morphogenesis by the Nodal pathway is an ancient trait that originated prior to the split of cnidarians and bilaterians [22]. Despite its conserved use throughout eumetazoans, there is a key difference in the site of expression and function of Nodal: it defines the left side in vertebrates and non-vertebrate chordates, but the right side in nonchordate deuterostomes and lophotrochozoans $[15,20]$. This change of expression is likely related to the proposed inversion of the dorso-ventral axis in the common ancestor of chordates [23-26], which caused a concomitant flipping of the right and left sides.

Amphioxus is advantageous for studying the events that occurred just after the dorso-ventral inversion. This group of marine invertebrates shares many common features (including notochord, dorsal nerve cord, pharyngeal gill slits, and metameric somital segments) with vertebrates, but lacks the vertebral column and the elaborate head structures derived from neural crest cells. Several vertebrate organs have identifiable homologs in amphioxus. The early developmental stages and adult stages of amphioxus are also highly similar to their counterparts in vertebrates. Upon sequencing the whole genome $[27,28]$, the phylogenetic positioning of amphioxus at the earliest diverging chordate clade is supported, while vertebrates and the highly derived tunicates are now placed together as a sister group [29,30]. Its phylogenetic position among the chordates and similarities to vertebrates have enabled amphioxus to provide crucial insights into the ancestral state of vertebrate traits [31].

Amphioxus LR asymmetry is a peculiarity among other chordates and represents an interesting area of study (Figure 1). During embryonic development, the somites are formed asymmetrically on the left and right sides [32]; furthermore, the arrangement of somites is staggered, with the left set of somites positioned slightly forward as compared to the right set (Figure 1A,B). As a consequence, in larval and adult amphioxus, the muscle segments and peripheral nerves running along the myomere boundaries are out of register, with the left side positioned half a segment anterior to the right side [33] (Figure 1C). Even more conspicuously, the entire pharyngeal region displays a marked asymmetry (Figure 1D,E,F,G,H,I,J). The left-sided positioning of the mouth during the larval stages is considered unique to amphioxus (Figure $1 \mathrm{~F}$ ) and raises serious questions regarding the proposed homology with the median mouths of other chordates. On the left side, the structure related to the vertebrate anterior pituitary, the preoral pit, develops from the left coelomic pouch that fuses with the epidermis anterior to the mouth [34] (Figure 1E,G). On the right side, the pharyngeal wall differentiates into the endostyle (Figure 1E,H) that represents the homolog of the vertebrate thyroid gland and into the club-shaped gland, an enigmatic structure with no clear counterpart in other animals. The club-shaped gland forms a transverse tube, the dorsal secretory part of which connects to the right pharyngeal wall, while the ventral non-secretory duct opens externally on the left side [35] (Figure 1E,H,I). The pharyngeal asymmetry is further exaggerated by the first few gill slits that develop ventrally on the right side behind the club-shaped gland (Figure 1E,J). Although the marked LR asymmetric features of amphioxus have been known since the late 19th century (reviewed in [1]), it is still not completely clear whether this asymmetry is also controlled by the conserved Nodal signaling cascade. During the last decade, the expression patterns of homologs of vertebrate Nodal, Lefty2, Gdf1, Cerl2, and Pitx2 have been described, and the LR asymmetric expression patterns of those genes hint that Nodal signaling may play a role in patterning the LR axis in amphioxus [36-41]. However, functional studies of the Nodal pathway during LR patterning have not been carried out in the amphioxus system to date.

In this study, we describe asymmetric expression of members of the Nodal signaling cascade and other putative downstream factors that are likely involved in establishing the LR asymmetry of the amphioxus body. Following the application of chemical inhibitors against Nodal signaling during amphioxus embryogenesis, we observed loss of expression of left-sided genes and ectopic expression of right-sided genes on the left side. Upon Nodal inhibition, the larvae exhibit a bilaterally symmetrical phenotype with duplication of the right-sided structures and suppression of left-sided morphology, involving loss of the mouth opening and preoral pit and loss of the offset arrangement of paraxial structures. Our findings indicate that Nodal signaling is necessary for the induction of asymmetric expression of downstream genes and for the formation of profound asymmetry of the amphioxus body. 

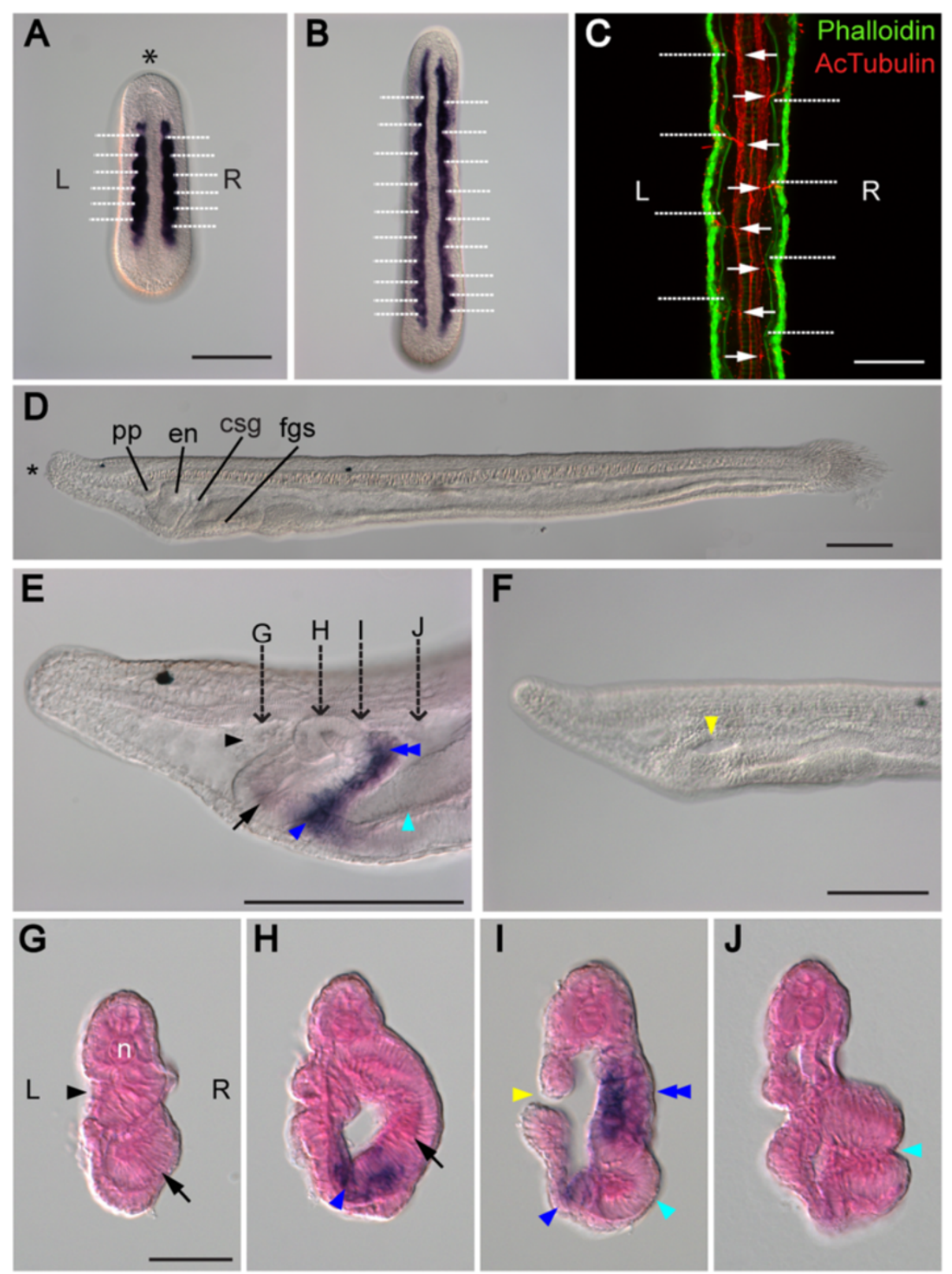

Figure 1 The left-right asymmetric characters of amphioxus. (A, B) Dorsal views of mid-neurula (N2) and late neurula (N3), marked with $m$-actin to display asymmetric arrangement of somites. White dotted lines mark the boundary of each somital segment. The asterisk $\left(^{*}\right)$ marks the anterior; ' $L$ ' represents the left side and ' $R$ ' the right side of the embryo. Scale bar, $100 \mu m$. (C) The myomeres (phalloidin staining) and axon bundles (acetylated a-tubulin staining) of amphioxus larva (L2) are positioned asymmetrically. White dotted lines mark myomere boundaries, and white arrows mark connections of peripheral axon bundles to the neural tube. Scale bar, $50 \mu \mathrm{m}$. (D) Left lateral view of L2-stage larva. Scale bar, $100 \mu \mathrm{m}$. en, endostyle; csg, club-shaped gland; fgs, first gill slit; pp, preoral pit. (E) Left lateral view of L2-stage larva focused on the right side. The club-shaped gland is marked with FoxE4 riboprobe. Dashed arrows mark the section planes in (G-J). Pharyngeal organs are labeled as described for G-J. Scale bar, $100 \mu \mathrm{m}$. (F) Left lateral view of L2-stage larva focused on the left-sided mouth opening (yellow arrowhead). Scale bar, $100 \mu \mathrm{m}$. (G-J) Sections of L2-stage larva from (E) showing asymmetrical positioning of pharyngeal organs. ' $n$ ' marks the notochord and the sections are seen from the posterior direction. The preoral pit (G, black arrowhead) and the mouth (I, yellow arrowhead) open on the left. The endostyle ( $\mathrm{H}$, black arrow) forms on the right. The dorsal part of the club-shaped gland (I, blue double arrowhead) is situated on the right, while its ventral part (duct of the club-shaped gland) extends to the left side ( $\mathrm{H}$, I, blue arrowhead). The first gill slit (I, J, cyan arrowhead) opens to the right side. Each section is $10 \mu \mathrm{m}$ thick. Scale bar, $25 \mu \mathrm{m}$. 


\section{Methods \\ Identification of left-right asymmetric gene markers in amphioxus}

The sequences of Branchiostoma floridae genes reported to be asymmetrically expressed along the left and right sides (including Gdf1/3 [38], Pitx [36,39], Nkx2.1 [42], Hand [43], m-actin [44], and Hu/Elav [33,45]) were BLAST (Basic Logical Alignment Search Tool)-searched against the $B$. floridae expressed sequence tag (EST) database [46] (http://amphioxus.icob.sinica.edu.tw/) to identify potential cDNA clones (Additional file 1: Table S1). The clones were amplified using EST-specific primers (Additional file 2: Table S2) to synthesize probes for RNA in situ hybridization. Clones of Nodal, Lefty, and FoxE4 were described previously $[40,41,47]$. B. floridae Cerberus cDNA, which could not be identified in the EST database, was amplified by polymerase chain reaction (PCR) using a cDNA library constructed in the pBluescript vector [48]. PCR was performed using the Expand High Fidelity ${ }^{\text {PLUS }}$ PCR System (Roche, Basel, Switzerland). PCR products were then ligated into the $\mathrm{pGEM}^{\circledR}-\mathrm{T}$ Easy vector (Promega, Madison, WI, USA), amplified, and sequenced.

Published sequences of amphioxus genes that were previously shown to display asymmetric expression in the pharynx (Pitx, Lhx3, Dkk1/2/4, FoxE4, FoxQ1, and Nkx2.1 (TTF-1) [36,39,42,47,49-53]) were BLAST-searched for their potential Branchiostoma lanceolatum orthologs using the European amphioxus transcriptome database [54]. After confirmation using translated nucleotide BLAST (tBLASTx), the candidate sequences were amplified using AccuPrime $P f x$ DNA Polymerase (Life Technologies, Carlsbad, CA, USA) and sequencespecific primers (see Additional file 2: Table S2). The PCR products were cloned into the pCR-Blunt II-TOPO vector (Life Technologies, Carlsbad, CA, USA), and the identity of the clones was confirmed by sequencing. The $B$. lanceolatum Krox clone was kindly provided by Stephanie Bertrand (Laboratoire Arago, Banyuls-sur-Mer, France).

\section{Animal collection}

Three species of amphioxus, Branchiostoma floridae, Branchiostoma belcheri, and Branchiostoma lanceolatum, were used in this study. B. floridae adults were collected in Tampa Bay, FL, USA, during the summer breeding season. $B$. belcheri adults were collected from Kinman Island near the Xiamen area in southeastern China [55]. Gametes were obtained by electric stimulation [56] or by spontaneous spawning of gravid animals. Fertilization and culturing of the embryos were carried out as previously described [56]. B. lanceolatum adults were collected in Banyuls-sur-Mer, France, prior to the summer breeding season and raised in the lab until spawning. The spawning of males and females was induced by temperature shift as described [57]. Embryos were staged according to Hirakow and Kajita [58,59], and neurula-stage embryos were further divided into more defined stages according to $\mathrm{Lu}$ et al. [33].

\section{In situ hybridization, immunostaining, cryosection, and image acquisition}

To synthesize riboprobes, cDNA fragments were amplified as templates as previously described [60]. Antisense or sense digoxigenin (DIG)-labeled riboprobes were synthesized using DIG RNA labeling mix (Roche, Basel, Switzerland) with T7 or SP6 RNA polymerase (Promega, Madison, WI, USA), depending on the insert orientation. Whole-mount in situ hybridization on amphioxus embryos was performed as previously described [61] with slight modifications to improve the results. Probe incubation during the hybridization process was performed at $65^{\circ} \mathrm{C}$ overnight. Immunostaining of F-actin and acetylated alpha-tubulin was carried out as previously described [33], with slight modifications. Embryos were de-ciliated by using a p1000 pipette to gently pipette seawater onto embryos placed in a $45-\mu \mathrm{m}$ meshed basket. The streaming seawater pushes the embryo against the meshed surface repeatedly, which creates a force that makes the surface cilia fall off. After this procedure, embryos were fixed with $4 \%$ of PFA in MOPS buffer. The secondary antibody used to detect acetylated alpha tubulin was changed to Alexa-635-conjugated goat anti-mouse antibody. Hoechst 33342 (Invitrogen, Carlsbad, CA, USA) was used for nuclear staining. Cryosections were obtained using a Leica CM1950 cryostat (Leica Biosystems, Heidelberg, Germany) after in situ hybridization.

Images of embryos were taken using a Zeiss Axio Imager A1 microscope with a Zeiss AxioCam MRc CCD camera (Carl Zeiss, Jena, Germany) or using a Nikon Diaphot 300 microscope (Nikon Corporation, Chiyoda, Tokyo, Japan) with a Canon EOS 1100D camera (Canon, Inc., Chichibu, Saitama, Japan). Immunofluorescence images were taken with a Leica TCS-SP5-AOBS confocal microscope (Leica Microsystems, Wetzlar, Germany). Image and Leica LAS AF were used to minimally adjust the brightness of photographs and to construct montage images of the whole larvae from multiple photographs. Imaris x64 program (version 8.0.1) was used to produce $3 \mathrm{D}$ reconstructions of the pharyngeal morphology of $B$. floridae larvae, with Hoechst 33342 nuclear staining acting as the template. Z-sections (section thickness: $1.968 \mu \mathrm{m}$; stepsize: $0.8 \mu \mathrm{m}$ ) of larvae were imaged from the dorsal side; a Leica TCS-SP5-AOBS multiphoton beam with an excitation wavelength of $810 \mathrm{~nm}$ was used to increase the resolution of each z-stack. Overall larval morphology was created using the automated function, with the nuclear signal on each layer acting as the template. Whole mount larval morphology was then turned transparent, while the pharyngeal region was selected 
out by manipulating the intensity threshold and voxel value. The preoral pit, endostyle, club-shaped gland, and gill slits were contoured manually by selecting the outline of the nuclear staining of each organ across each zsection from the dorso-ventral plane. To ensure that the manually contoured structures accurately depicted the relevant organs, an automated contour of the whole pharyngeal region was generated; the automatic and manual contours were cross-checked to ensure they overlapped. Further cross-checking with previous cryosections was conducted to ensure consistency of the model. The outline of the mouth was generated by removing the rest of the automated pharyngeal region model with several clipping planes, leaving the surface around the mouth opening. Snapshots and videos of the 3D model were also made in Imaris x64.

\section{Pharmacological inhibition of Nodal signaling}

B. floridae, B. belcheri, and B. lanceolatum embryos and larvae were treated with Nodal signaling inhibitors SB431542 (Tocris, Bristol, UK) and SB505124 (Sigma, St. Louis, $\mathrm{MO}$, USA) at the indicated concentrations and for various lengths of time.

For B. floridae and B. belcheri treatments, SB505124 and SB431542 were dissolved in dimethyl sulfoxide (DMSO) to prepare a $50 \mathrm{mM}$ stock. The stock was then diluted with filtered seawater to make working solutions of 5 and $10 \mu \mathrm{M}$ for SB505124 and 10 and $20 \mu \mathrm{M}$ for SB431542. The embryos were treated at the late gastrula stage (G5/6) by direct application. The control group was treated with equal amounts of DMSO. The embryos were then collected and fixed at early pre-hatching neurula (N0), early neurula (N1), mid-neurula (N2), late neurula (N3), and open mouth (L2) stages for further analysis.

For B. lanceolatum treatments, SB505124 was dissolved in DMSO to prepare a $3 \mathrm{mM}$ stock. This stock solution was diluted in filtered seawater to final concentrations of $0.1,0.5,1.0,5,10$, and $50 \mu \mathrm{M}$ and applied to amphioxus embryos at N0. The larvae were raised until the open mouth stage and then fixed. Control embryos were treated with filtered seawater containing an equal amount of DMSO. Larvae treated with a concentration of $0.1 \mu \mathrm{M}$ displayed a wild-type phenotype while larvae treated with $0.5 \mu \mathrm{M}$ or higher showed a phenotype with altered morphology. After this pilot experiment, we performed two types of treatments. For long-term treatments, embryos were raised in $1 \mu \mathrm{M}$ SB505124 from cap-shaped gastrula (G3), mid-gastrula (G4), late gastrula (G5/G6), gastrula/neurula (N0), hatching neurula (N1), mid-neurula (N2), or early larva (L1) until the open mouth stage (L2) and then fixed. For timerestricted treatments, embryos at G4, G5, N0, or N1 were treated with $0.5 \mu \mathrm{M}$ SB505124; the concentration was adjusted to $0.1 \mu \mathrm{M}$ by adding the adequate amount of filtered seawater at N0, N1, N2, N3, or L1. Embryos were then raised until L2 and fixed. After fixation, the control and treated larvae were used for morphological analysis and in situ hybridization.

\section{Results}

\section{Asymmetric expression of developmental regulatory genes across the left-right axis during amphioxus embryogenesis and larval development}

To establish whether cephalochordates use a conserved genetic network for LR patterning, we first surveyed the expression patterns of amphioxus homologs of the Nodal signaling cascade and genes known to be responsible for vertebrate LR asymmetric development. Previously, it has been shown that Nodal, Gdf1/3, Lefty, and Cerberus are all expressed symmetrically in the dorsal mesendoderm during amphioxus gastrulation [37,38,40,41]. To further determine the exact starting point of LR asymmetric expression of these important signaling molecules, we carefully selected finely staged $B$. floridae neurulae for examination. At the N0 stage (neurulae with zero somites), Nodal and Gdf1/3 are expressed bilaterally in the dorsal paraxial mesoderm (Figure 2A,B). Interestingly, Lefty expression becomes localized to the left side up to the embryonic midline (Figure 2C). On the other hand, the Nodal antagonist Cerberus is expressed only on the right side at this stage (Figure 2D). Thus, left-sided Lefty expression and right-sided Cerberus expression appear to represent the earliest recognizable LR asymmetric gene expression during amphioxus embryogenesis. In addition, Pitx expression cannot be detected in six of the ten N0stage embryos examined (Figure 2E); in the remaining four N0-stage embryos, Pitx is expressed weakly on the left side (Figure 2F), suggesting that left-sided Pitx expression has just begun to be established at the N0 stage.

At the N1 stage (neurulae with three somites), Nodal is still expressed in a bilateral fashion, but the expression in the left domain is stronger than that in the right domain (Figure 2G). Similarly, the expression domains of Gdf1/3 and Lefty are more restricted to the left side by this stage (Figure 2H,I); Lefty transcripts are also found medially up to the embryonic midline (Figure 2I, white arrowhead). Cerberus is expressed on the right side of the embryo (Figure 2J), and some Cerberus transcripts can be detected at the midline (Figure 2J, white arrowhead). At this stage, Pitx is strongly expressed on the left side in both the mesendoderm and the ectoderm (Figure 2K).

At the N2 stage (neurulae with eight somites), Nodal, Gdf1/3, and Lefty transcripts are found mainly on the left side (Figure 2L,M,N). Cerberus expression is no longer observed at the right side, but instead is located in the midline (Figure 2O). Pitx expression remains on the left side in the anterior region of the embryo (Figure 2P). 


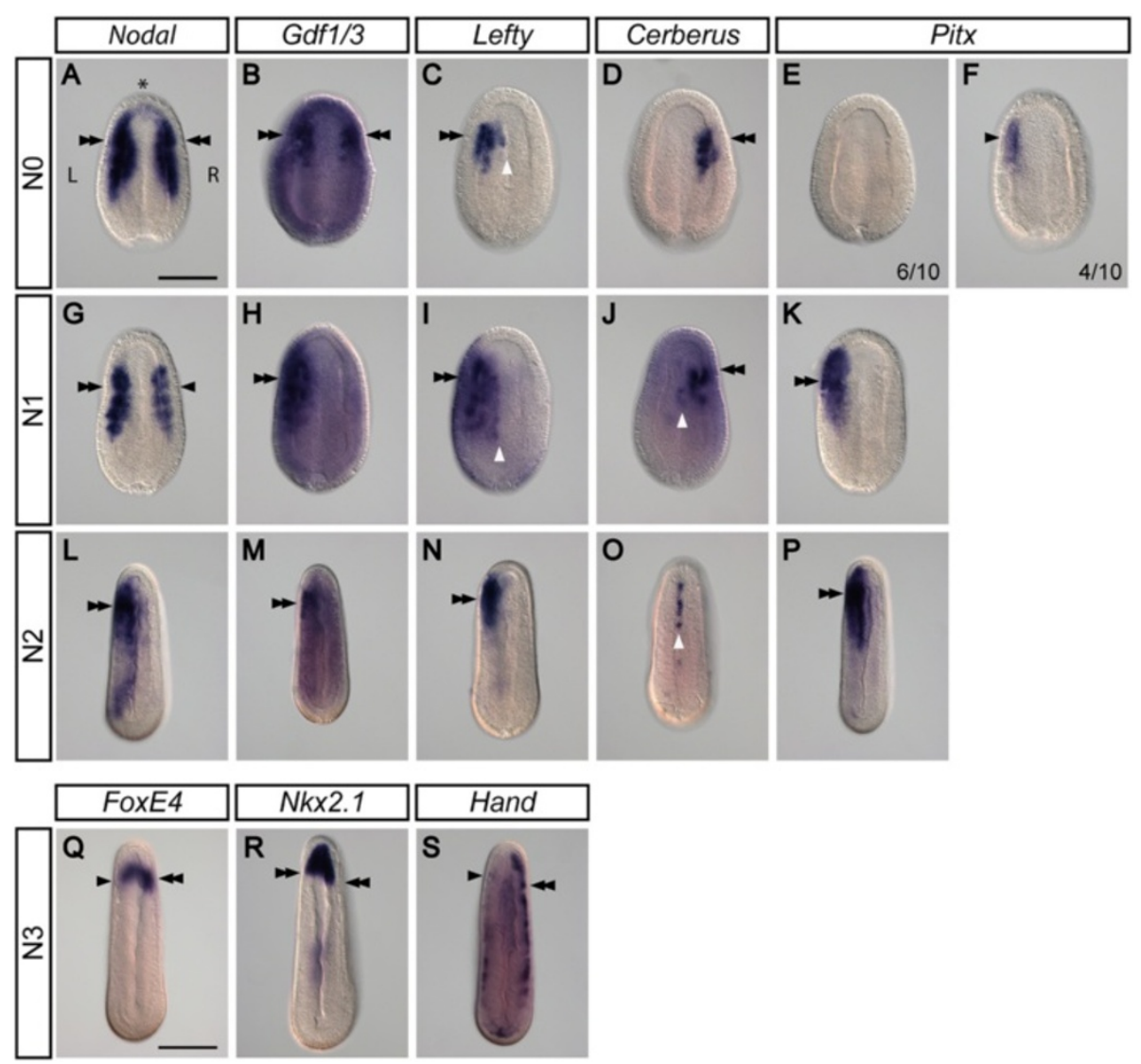

Figure $\mathbf{2}$ Left-right asymmetric expression of developmental regulatory genes during amphioxus embryogenesis. All images are of $B$. floridae, taken from the dorsal view. ' $L$ ' marks the left side, ' $R$ ' marks the right side, and the asterisk (*) marks the anterior. Double arrowheads indicate stronger expression patterns, and single arrowheads indicate relatively weaker expression patterns. White arrowheads indicate the midline. Scale bar, $100 \mu \mathrm{m}$. (A-F) At the onset of neurulation (stage N0), Nodal and Gdf1/3 are expressed symmetrically on both the left and the right sides of the embryo. Lefty is expressed on the left side, while Cerberus is expressed on the right side; these are the first signs of asymmetrical gene regulation. Pitx is expressed on the left side of a fraction of embryos, suggesting that Pitx is just starting to be transcribed. (G-K) At the early neurula stage (N1), Nodal becomes expressed preferentially on the left side, while Gdf1/3, Lefty, and Pitx are expressed exclusively on the left side. Additionally, Lefty is also expressed at the midline. Cerberus transcripts are found on the right side and also at the midline. (L-P) At the midneurula stage (N2), Nodal, Gdf1/3, Lefty, and Pitx are expressed exclusively on the left side while Cerberus is expressed at the midline. (Q-S) At the late neurula stage (N3), some organ-specific genes already display asymmetric expression. Expression of FoxE4, Nkx2.1, and Hand is biased towards the right side.

In addition to the aforementioned Nodal cascade genes, several transcription factor genes also display the LR asymmetric expression pattern during this stage and the subsequent N3 stage (neurulae with more than eight somites). FoxE4, a gene specifically expressed in the developing club-shaped gland and the adult endostyle $[47,62]$, is preferentially expressed on the right side (Figure $2 \mathrm{Q}$ ). $N k \times 2.1$ is expressed in the anterior archenteron, with right-sided expression reaching slightly posterior to the expression on the left side (Figure 2R). The Hand gene is expressed in the lateral/ventral mesoderm during amphioxus development [43], and its expression is consistently stronger on the right side as compared to that on the left side (Figure 2S).

Major organogenesis starts to take place during the subsequent larval stages. At the L1 stage, the larva exhibits LR asymmetric expression of several genes in the pharyngeal region. Here, we show B. lanceolatum images as representatives in Figure 3, because during the course of this study, we obtained more pharyngeal gene markers from B. lanceolatum. Available gene expression patterns from $B$. floridae show identical patterns (Additional file 3: Figure S1). On the left side, Pitx is expressed broadly in the anterior pharynx spanning the mouth and preoral pit areas (Figure $3 \mathrm{~A}, \mathrm{~A}^{\prime}$ ), whereas $L h x 3$ and $D k k 1 / 2 / 4$ expression is more focused (Figure 3B,B,C, $\left.C^{\prime}\right)$. Aside from their expression in the neural tube, $L h x 3$ transcripts are also found in the prospective preoral pit, while $D k k 1 / 2 / 4$ is expressed superficially both in the prospective preoral pit and in the region destined to develop into the mouth opening (Figure $3 \mathrm{~B}, \mathrm{~B}, \mathrm{C}, \mathrm{C}^{\prime}$ ). On the right side, FoxE4 and Krox are both expressed just opposite the 


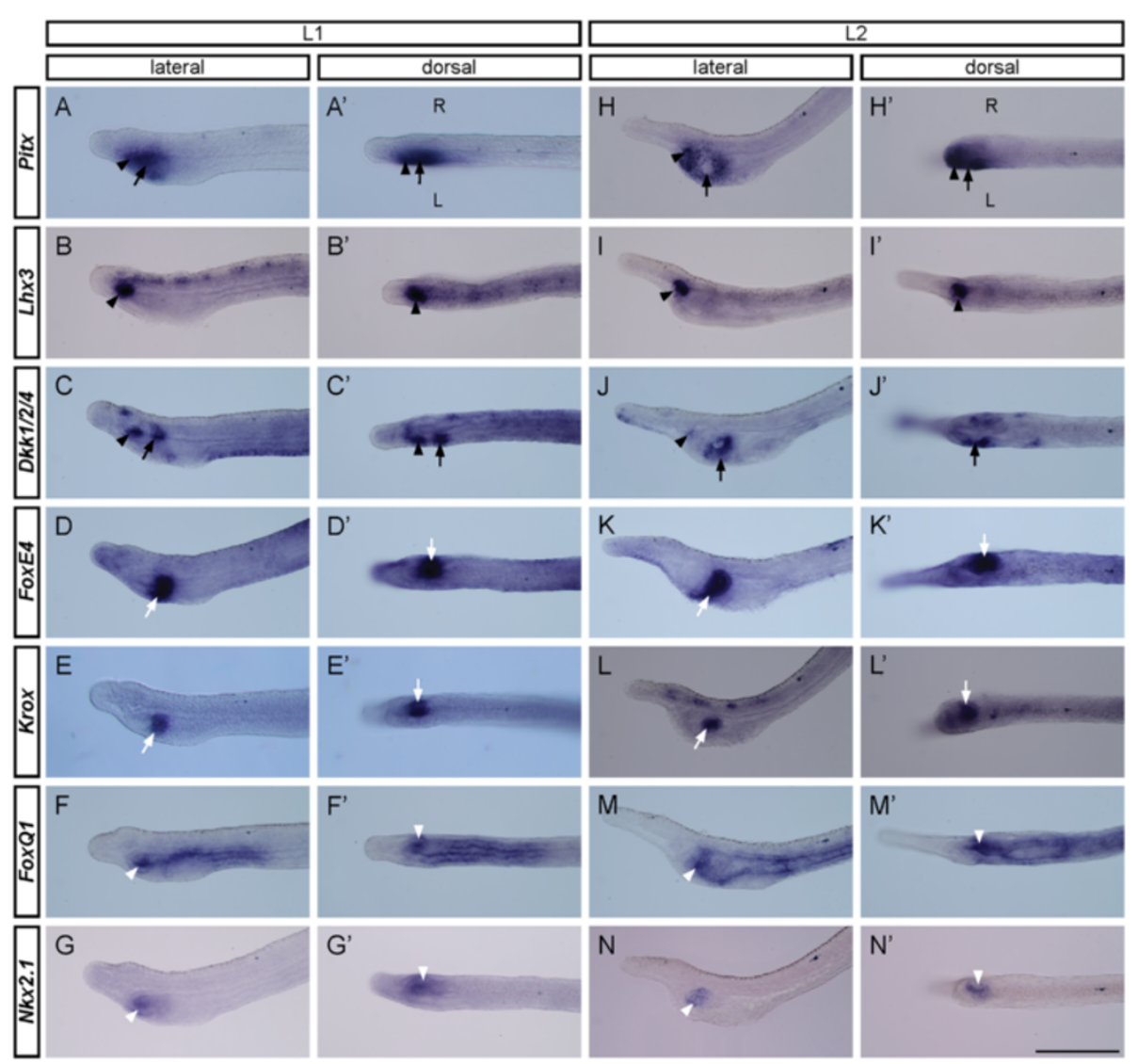

Figure 3 Left-right asymmetric expression of developmental regulatory genes during larval stages. Images are of $B$. lanceolatum at the early larval stage (L1) and the open mouth stage (L2) from either left lateral view or dorsal view. ' $L$ ' marks the left side and ' $\mathrm{R}$ ' marks the right side; anterior is to the left. Black arrowheads indicate the preoral pit, black arrows mark the mouth region, white arrows point to the club-shaped gland, and white arrowheads mark the endostyle. Scale bar, $100 \mu \mathrm{m}$. (A-G, $\mathbf{A}^{\prime}-\mathbf{G}^{\prime}$ ) At L1, Pitx is expressed broadly in the left anterior pharyngeal region spanning the area of the prospective preoral pit and the mouth. $L h \times 3$ expression is confined to the prospective preoral pit, while $D k k 1 / 2 / 4$ is expressed focally in the regions destined to form both the preoral pit and the mouth. FoxE4 and Krox transcripts are restricted to the prospective club-shaped gland, where the expression pattern of Krox is nested within that of FoxE4. FoxQ1 is expressed anterior to FoxE4 in the prospective endostyle region and also at other regions within the pharynx. Nkx2.1 transcripts are confined solely to the endostyle region. ( $\mathbf{H}^{\left.-\mathbf{N}, \mathbf{H}^{\prime}-\mathbf{N} \mathbf{N}^{\prime}\right)}$ The L2 stage exhibits well-developed pharyngeal structures, with expression patterns similar to those at L1. Pitx and Dkk1/2/4 continue to be expressed in the preoral pit and the mouth, while $L h x 3$ expression is confined solely to the preoral pit. FoxE4 and Krox are expressed in the club-shaped gland, with FoxE4 marking the whole structure, while Krox transcripts are confined to its dorsal part. In addition to its expression elsewhere in the pharynx, FoxQ1 is co-expressed with NkX2.1 in the endostyle.

prospective mouth opening, with the Krox pattern nested within the FoxE4 pattern (Figure 3D,D',E,E'). Nkx2.1 and FoxQ1 are expressed slightly anterior to the FoxE4-Krox pattern on the right side; FoxQ1 is also expressed posteriorly along the length of the pharynx (Figure 3F,F,G,G').

At the L2 stage, the pharyngeal region differentiates into distinct organs, namely the preoral pit and mouth on the left side and the endostyle and club-shaped gland on the right side. On the left side, Pitx and Dkk1/2/4 continue to be expressed at the margins of the mouth opening and, together with $L h x 3$, are also expressed in the preoral pit (Figure 3H,I,J,H,',','). On the right side, FoxE4 transcripts become confined to the whole clubshaped gland (Figure $3 \mathrm{~K}, \mathrm{~K}^{\prime}$ ), while Krox is confined to the dorsal part of this gland (Figure 3L,L'). Additionally, expression of both $N k \times 2.1$ and FoxQ1 is largely restricted to the endostyle on the right side, and FoxQ1 expression is also observed in the pharyngeal bands (Figure 3M,M'N,N').

In summary, we observed gradual establishment of asymmetric gene expression of (i) signaling molecules in the Nodal signaling cascade and (ii) the putative downstream transcription factor Pitx across the LR axis during the early neurula stage ( $\mathrm{N} 0$ to $\mathrm{N} 1$ ). We also characterized a number of marker genes that exhibited LR asymmetric expression patterns in the mid/late neurula stage or in the subsequent larval stages. In the following sections, we will use the expression patterns of 

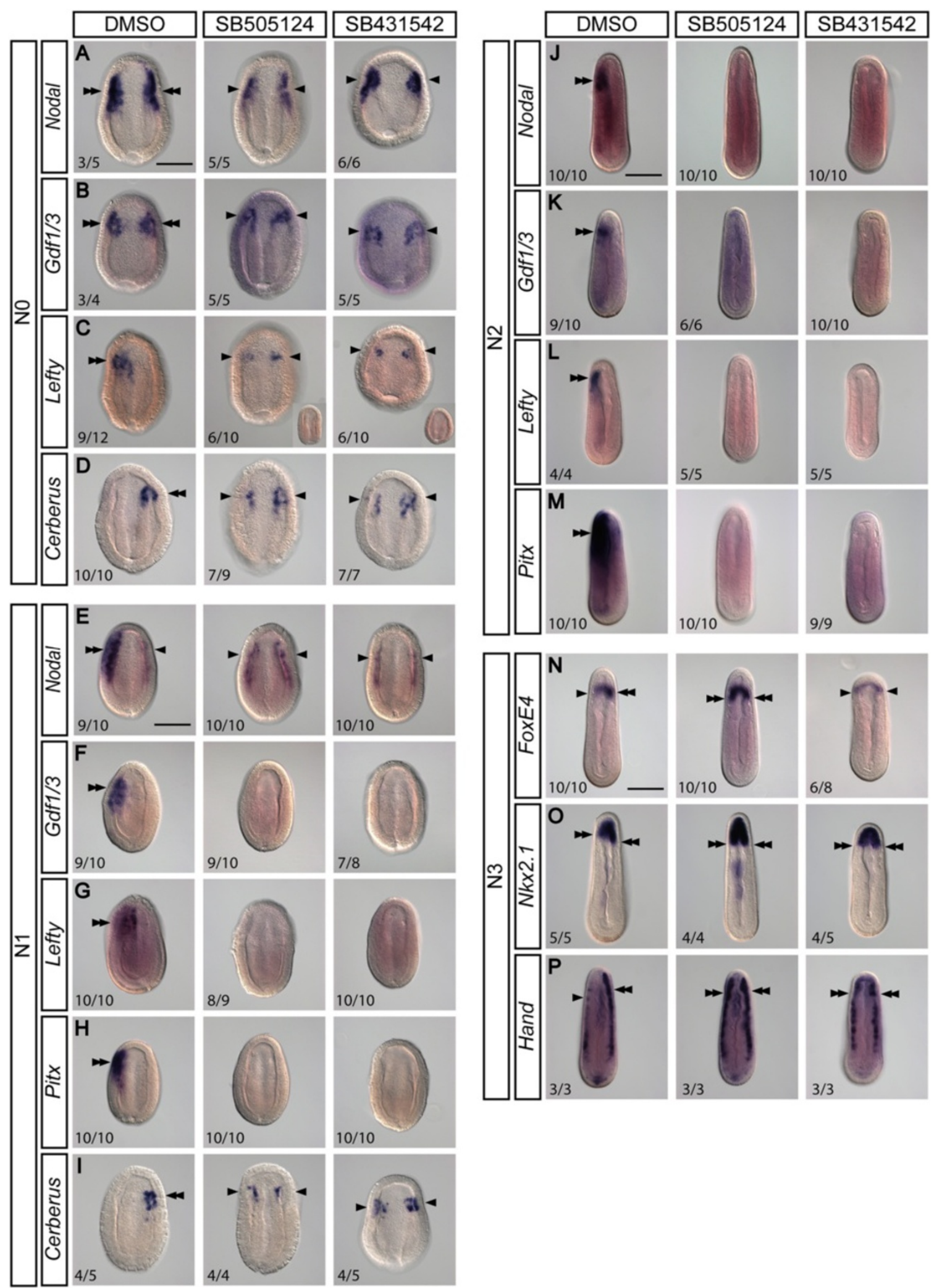

Figure 4 (See legend on next page.) 
(See figure on previous page.)

Figure 4 Inhibition of the Nodal signaling pathway changes the expression pattern of left-right regulatory genes. Images are of $B$. floridae taken from the dorsal view. Double arrowheads mark strong expression and single arrowheads mark weak expression. Scale bar, $100 \mu$ m. (A-D) At the onset of neurulation (N0), embryos treated with Nodal inhibitors exhibit bilateral expression of Nodal and Gdf1/3, albeit diminished as compared to controls. Similarly, the strong unilateral expression of Lefty and Cerberus in controls is converted into reduced bilateral expression by each treatment. (E-I) At the early neurula stage (N1), Nodal expression remains bilateral but is greatly diminished in the treated embryos. Expression of genes expressed on the left side (Gdf1/3, Lefty, and Pitx) is lost in the treated embryos, while Cerberus expression becomes bilaterally symmetrical. (J-M) At the mid-neurula stage (N2), Nodal, Gdf1/3, Lefty, and Pitx expression cannot be detected in the treated embryos. (N-P) At the late neurula stage (N3), the right-sided bias in expression of FoxE4, Nkx2.1, and Hand is abolished by the treatment, and these genes become symmetrically expressed on both left and right sides.

these marker genes to assess the effects of blocking Nodal signaling with small molecule inhibitors during amphioxus development.

\section{Treatments with Nodal signaling inhibitors disturb the expression of early left-right regulatory genes, even before morphological asymmetry can be observed}

We next examined whether blocking Nodal signaling would affect the asymmetric expression of Nodal cascade genes and other LR marker genes. We treated $B$. floridae embryos with Nodal signaling inhibitors SB505124 and SB431542 starting from the late gastrula stage (G5/G6) and then analyzed these embryos at subsequent stages. Treated N0 embryos exhibit bilateral expression of Nodal and Gdf1/3, as compared to controls (Figure 4A,B). Expression of Lefty and Cerberus, which is restricted to either the left or right sides in control embryos, becomes bilateral for both genes upon the treatment (Figure 4C,D). The treated larvae exhibit slightly reduced expression levels of all the examined factors, and in the case of Lefty, the expression can even be reduced to an undetectable level (Figure 4C, inset images).

At the N1 stage, when expression of Nodal is normally biased to the left side, the treated embryos display weak Nodal expression on both sides, and the pattern appears to be symmetrical (Figure 4E). Similarly, expression of the other two left-sided genes, Gdf1/3 and Lefty, is abolished by the treatment (Figure 4F,G) and expression of the downstream transcription factor Pitx is also lost (Figure $4 \mathrm{H}$ ). On the other hand, treatment changes the expression pattern of Cerberus from right-sided to bilateral (Figure 4I). At the subsequent N2 stage, treatment abolishes expression of Nodal (Figure 4J). Similarly, expression of Gdf1/3, Lefty, and Pitx is no longer detectable in the treated N2 embryos (Figure 4K,L,M).

Analysis of other genes displaying asymmetric expression at N3 revealed that their expression patterns are also affected by treatments with Nodal inhibitors. FoxE4, the expression of which is biased towards the right pharyngeal wall in wild types, exhibits symmetric expression in a horseshoe-shaped pattern upon either treatment (Figure $4 \mathrm{~N}$ ). In a similar manner, the rightward-biased expression pattern of $N k x 2.1$ becomes symmetrical following treatment (Figure 4O); the expression of
Hand, which normally shows differential expression between the left and right lateral plate mesoderm, becomes equally strong on both sides (Figure 4P).

Taken together, these data demonstrate that inhibition of Nodal signaling abolishes the asymmetrical expression of the Nodal cascade genes and results in symmetrical expression of other downstream organ-specific genes during the neurula stage; therefore, Nodal signaling is necessary for establishing the LR molecular asymmetry in developing amphioxus embryos.

\section{Treatments with Nodal signaling inhibitors alter the} left-right asymmetric arrangement of developing muscle blocks and the nervous system

Asymmetrical somite arrangement, which occurs at the mid-neurula stage, is the earliest recognizable morphological feature of LR asymmetric development in amphioxus (reviewed in [1]). To investigate the effect of blocking Nodal signaling on the development of somite structures, we treated $B$. floridae and B. belcheri embryos with SB505124 and SB431542 from the late gastrula stage (G5/6) and then observed somite development in the neurula and myomere structure at larval stages using phalloidin staining of F-actin or using in situ hybridization of the muscle actin gene (m-actin). Phalloidin staining enabled detection of mild asymmetrical arrangement of somites in N2-stage neurulae, with left-sided somites positioned slightly more forward as compared to those on the right (Figure 5A, DMSO control); this asymmetrical pattern became more apparent at the N3 stage when embryos have more than eight somites (Figure 5B, DMSO control). After treatments with either Nodal inhibitors, somites become symmetrically aligned on both sides of the developing notochord at the N2 and N3 stages (Figure 5A,B). Consistently, m-actin in situ hybridization showed the same effect of blocking Nodal signaling on somite asymmetry (Figure $5 \mathrm{C}$ ), that is, the $m$-actin-expressing muscle precursor cells became symmetrically aligned in the treated N3 embryos. This effect is especially apparent in the middle/posterior part of the body, where the normal asymmetric arrangement of somites is most easily discerned (Figure 5C, white dashed lines). 


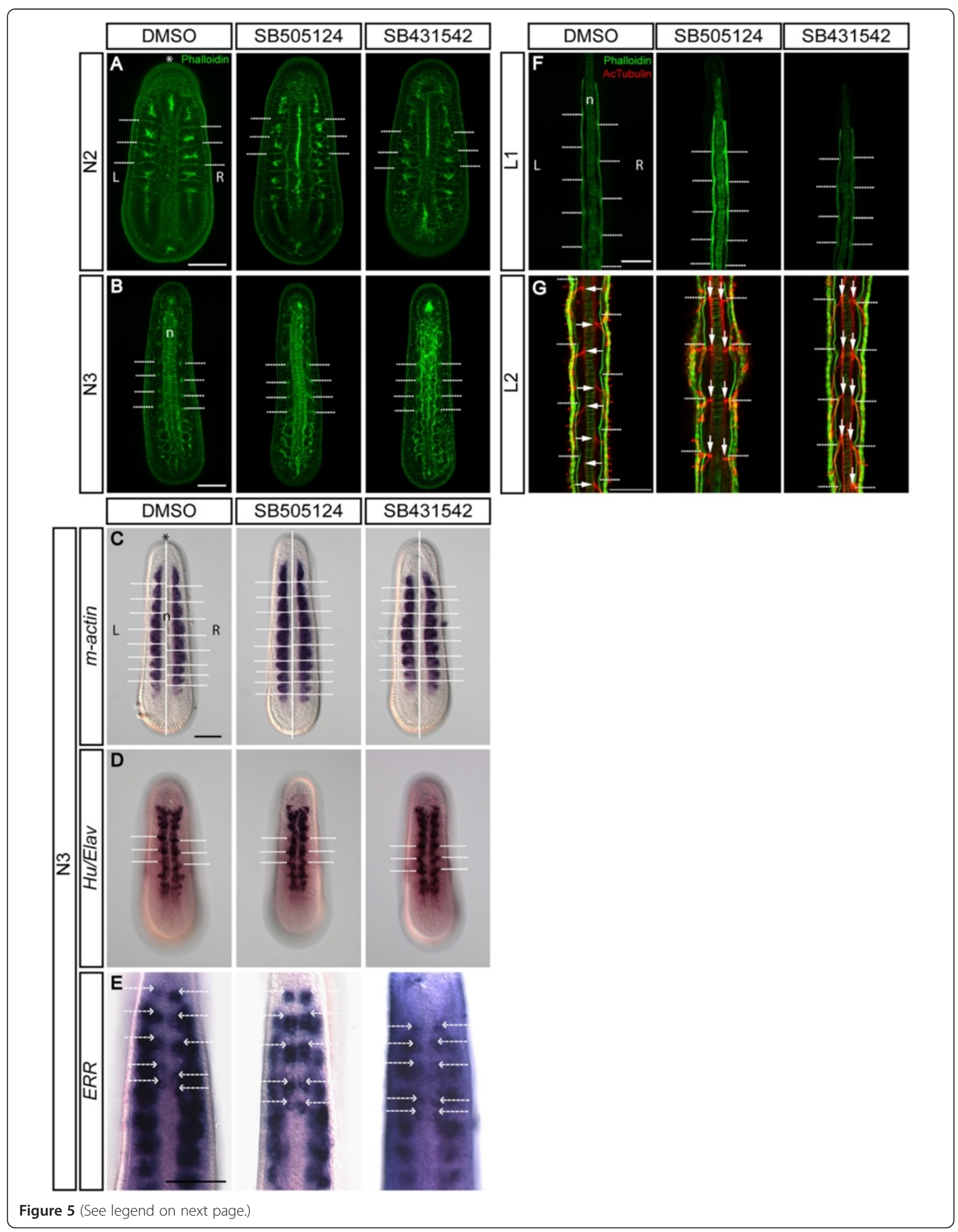




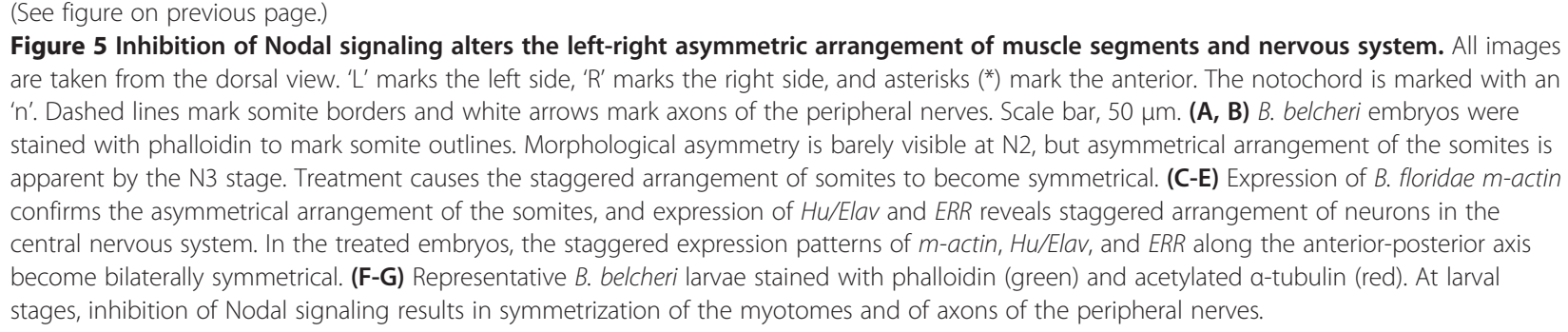

Previous anatomical studies have revealed the asymmetrical arrangement of amphioxus somatic motoneurons in the central nervous system (CNS) and their axonal tract structures [63-65]. Several studies on developmental gene expression patterns also uncovered such LR asymmetric patterns in putative CNS neurons during amphioxus embryogenesis [45,66-70], indicating that the developing CNS also exhibits LR asymmetry. More importantly, this CNS neuron asymmetry corresponds to the asymmetric somite arrangement in neurula-stage embryos, suggesting that their development might be controlled by a common developmental pathway. To determine whether Nodal signaling also controls the LR asymmetrical distribution of CNS neurons, we used the expression pattern of the pan-neuronal marker Hu/Elav to examine the distribution of post-mitotic neurons in the embryos treated with the two Nodal signaling inhibitors. Consistent with previous descriptions, we observed that CNS neurons form recognizable clusters in two longitudinal columns along the rostral-caudal axis of the developing CNS in normal N3-stage embryos (Figure 5D, DMSO control). Much like the asymmetry of somites, the arrangement of these neuronal clusters is staggered between the left and right columns, with the left ones being positioned slightly forward of the right ones (Figure 5D, white dashed lines in DMSO control). Nodal inhibitor treatments caused the arrangement of CNS neuronal clusters to become bilaterally symmetrical (Figure 5D), consistent with the change seen in the somite development. In addition, $E R R$, which stains both somites and certain neurons in the anterior CNS [66], also exhibits asymmetrical expression along the LR axis (Figure 5E, DMSO control). Upon treatment, expression of ERR loses its staggered arrangement along the anterior-posterior axis and becomes bilaterally symmetrical (Figure 5E). The arrangement of somatic musculature and neurons at the N3 stage becomes more pronounced with subsequent differentiation of these tissues; by L1 and L2, the control larvae exhibit a clear alternating pattern of muscle blocks and axonal structures of spinal nerves (Figure 5F,G, DMSO controls), while the larvae treated with Nodal inhibitors display bilaterally symmetrical patterns of muscle blocks and the axon-innervated positions of the CNS (Figure 5F,G).
Overall, these data suggest that the Nodal signaling pathway is necessary for the proper development of asymmetrical muscular and neural tissues in amphioxus and that inhibition of the Nodal pathway causes symmetrization of these tissues.

Inhibition of Nodal signaling results in the loss of the leftsided mouth opening and duplication of right-sided pharyngeal structures

To investigate the effect of inhibition of Nodal signaling on the development of the amphioxus pharynx, we treated B. lanceolatum and B. floridae embryos with SB505124 from N0 to L2. We analyzed the pharyngeal morphology of the control and treated larvae using immunofluorescence and in situ hybridization (Figure 6 and Additional file 3: Figure S1).

Confocal microscopy analysis revealed that the pharynx of the treated larvae exhibits symmetrical morphology, with loss of left-sided structures such as the mouth and the preoral pit. Moreover, the left side of the treated larvae seems to be a mirror image of the right side, with duplication of the otherwise right-sided endostyle and club-shaped gland (Figure 6A, $\mathrm{A}^{\prime}$ ) To confirm the identity of individual pharyngeal structures, we performed in situ hybridization against previously identified organ-specific marker genes (see Figure 3). In agreement with the morphological analysis, in situ hybridization demonstrated that genes expressed in the preoral pit and in the mouth in the control larvae (Pitx, Lhx3, and $D k k 1 / 2 / 4)$ become downregulated following treatment (Figure 6B,B',C,C,D,D'). On the other hand, Krox and FoxE4 transcripts, which mark the right-sided clubshaped gland in wild type, exhibit ectopic expression on the left side of the treated larvae, thus presenting a bilaterally symmetrical signal (Figure 6E,E,'F,F'). Detailed analysis uncovered that the FoxE4-positive and Krox-negative region of the club-shaped gland (that is, the duct of the club-shaped gland) is lost in the treated larvae, suggesting that only the dorsal part of the club-shaped gland is duplicated (Figure 6I,I',J,J'). Similarly, FoxQ1 and Nkx2.1, which are expressed in the right-sided endostyle in wild type, display bilaterally symmetrical signals in the anterior portion of the pharynx upon treatment (Figure 6G,G, $\mathrm{H}, \mathrm{H}$, $\left.\mathrm{K}, \mathrm{K}^{\prime}\right)$. To confirm the phenotype observed in whole 


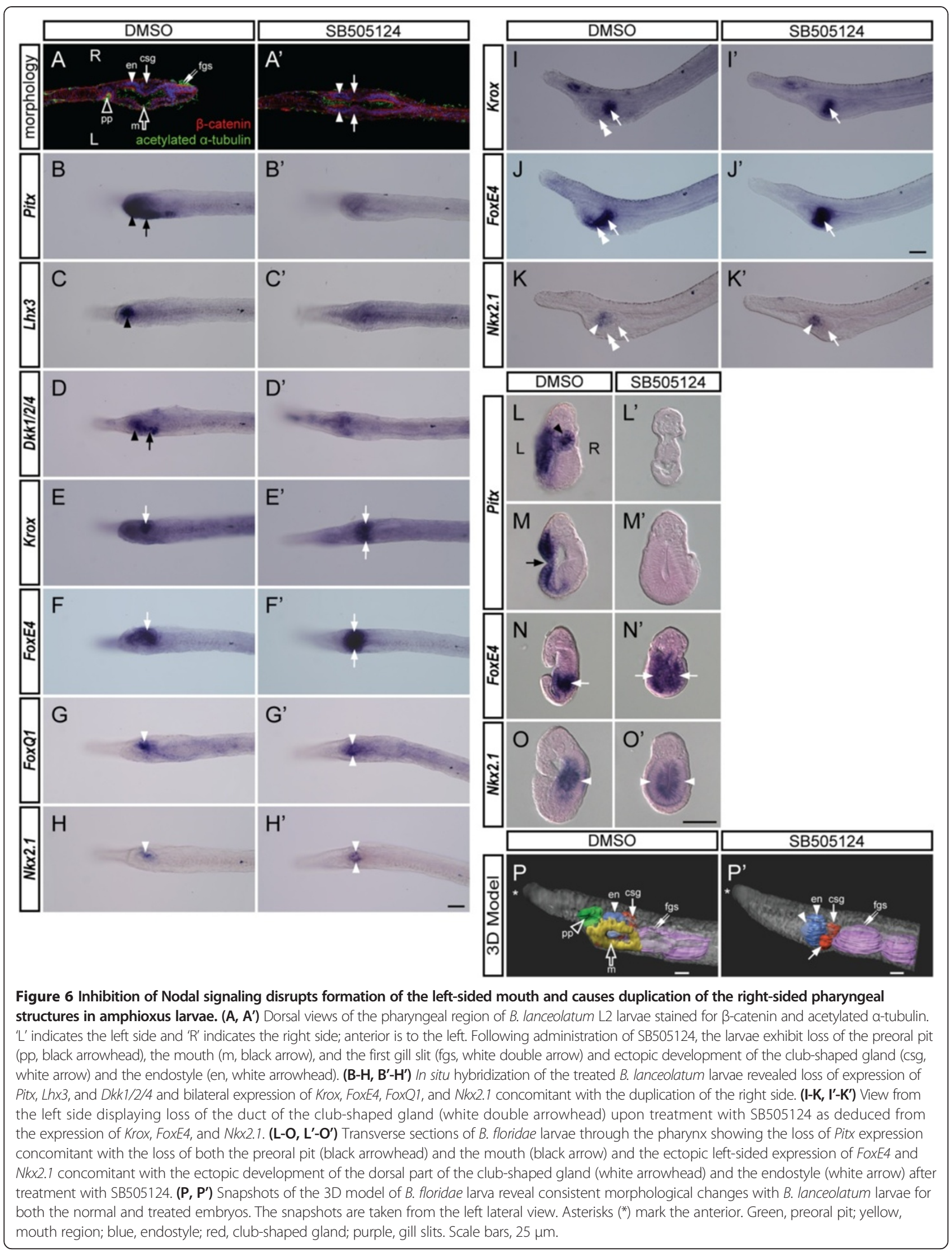


mounts, we sectioned the control and treated larvae upon hybridization. The treated larvae display loss of left-sided expression of Pitx concomitantly with loss of the mouth and preoral pit (Figure 6L,L'M,M'), and ectopic expression of FoxE4 and $N k \times 2.1$ on the left side, suggesting that the endostyle and the club-shaped gland develop on both the left and the right sides (Figure 6N,N,O,O'). To obtain more comprehensive images of the larval morphology, we used Imaris x64 to construct 3D organ models (Figure 6P,P', Additional file 4: Video S1, and Additional file 5: Video S2). Snapshots of the 3D model display consistent changes of morphology for the treated larvae. The predominantly leftsided preoral pit and mouth are lost upon treatment while the predominantly right-sided endostyle and club-shaped gland form ectopically on the left side, almost forming a mirror image of their right-sided counterparts.

These data suggest that inhibition of Nodal signaling results in the loss of left-sided identity, leading to the absence of the mouth and preoral pit and duplication of the right-sided identity, with ectopic development of the endostyle and club-shaped gland on the left side.

\section{Morphological asymmetry is specified during early neurula stages through asymmetrical expression of downstream targets}

We took advantage of the aforementioned system to determine the embryonic period during which LR asymmetry is specified. We performed a series of experiments in which SB505124 was applied to B. lanceolatum embryos for different time periods during development, and the resulting phenotypes at the open mouth stage (L2) were subsequently examined by in situ hybridization against the left-sided marker $L h x 3$ and the right-sided marker Krox. Phenotypes were divided into three categories (Figure 7A): 'wild type' (asymmetrical pharyngeal morphology; expression of both $L h x 3$ and Krox present), 'mild' (asymmetrical, but altered pharyngeal morphology; expression of $L h x 3$ reduced or absent, expression of Krox present on the right side), and 'strong' (symmetrical pharyngeal morphology; expression of Lhx3 lost, expression of Krox present on both left and right sides).

We first performed long-term treatments, in which SB505124 was administered to amphioxus embryos at

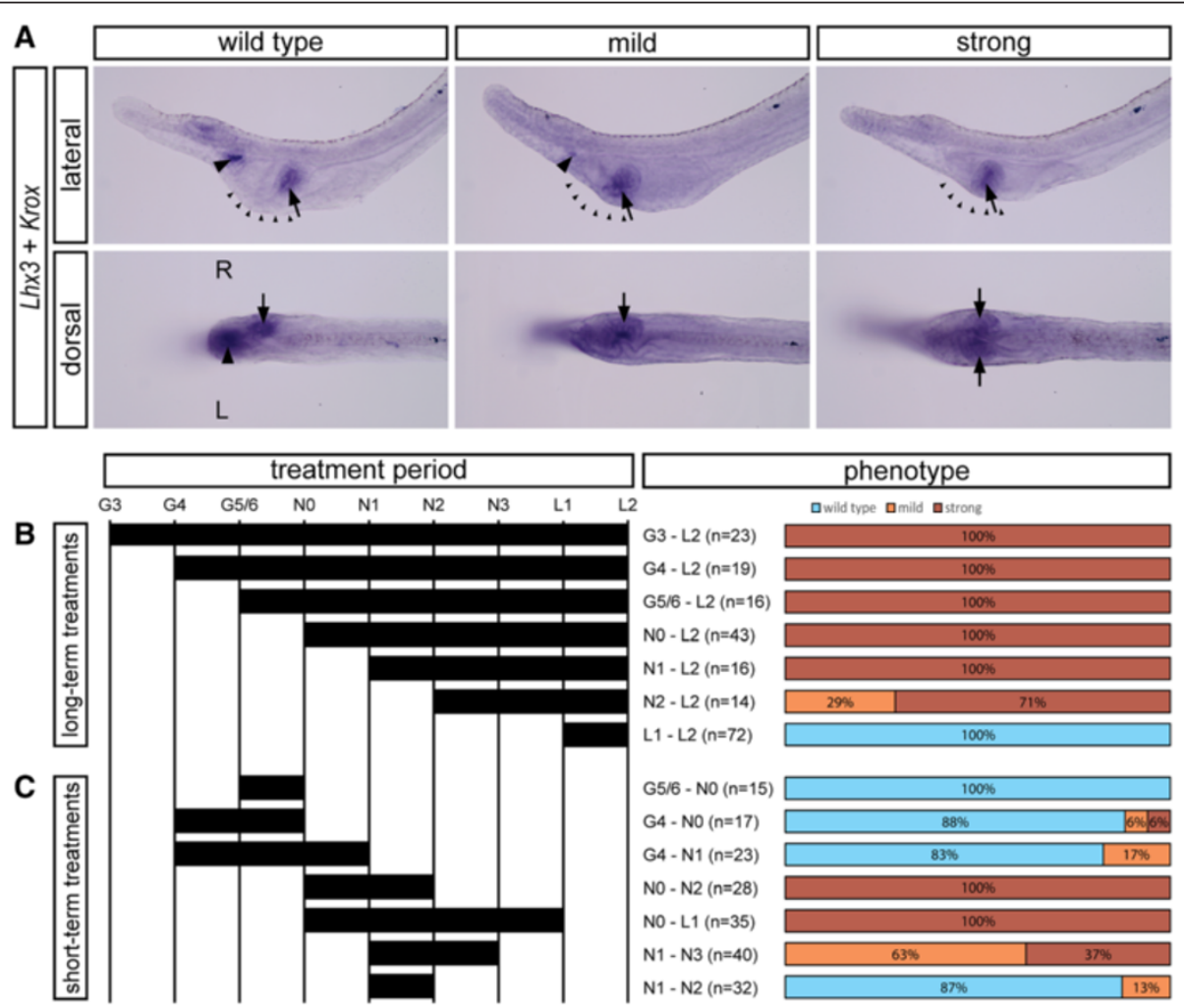

Figure 7 Time-restricted inhibition of Nodal signaling reveals that LR asymmetry is specified at neurula stages. (A) Inhibition of Nodal signaling by SB505124 results in three larval (L2) phenotypes, designated as 'wild-type', 'mild', and 'strong', and defined by differential expression of $L h \times 3$ as a marker of the preoral pit and Krox as a marker of the dorsal part of the club-shaped gland. The mild phenotype involves a loss of the ventral portion of the pharynx (small arrowheads) and reduced $L h \times 3$ expression, indicative of a reduced preoral pit (arrowhead). The strong phenotype involves a loss of the ventral portion of the pharynx (small arrowheads), complete loss of the preoral pit, and an ectopic club-shaped gland on the left side (arrow). (B) Long-term treatments were performed to demonstrate that Nodal inhibition during the neurula stages results in altered phenotypes. (C) Short-term treatments were performed to demonstrate that Nodal inhibition results in altered phenotypes, especially when administered at the N0 to N2 stages. All images are from B. lanceolatum. 
G3, G4, G5/6, N0, N1, N2, or L1 stages (Figure 7B). Application of the inhibitor at the N1 stage or earlier results in larvae with the strong phenotype, application at $\mathrm{N} 2$ to $\mathrm{L} 2$ results in larvae with either strong or mild phenotype, and application at L1 to L2 results in larvae with a wild-type phenotype. This suggests that larval morphology is most susceptible to inhibition during early neurula stages. To confirm this hypothesis, we also performed short-term treatments (Figure 7C). Application of the inhibitor during late gastrula stages does not result in a significant increase of altered morphology, and the majority of the larvae display a wild-type phenotype. On the other hand, application at early neurula stages, especially spanning N0 to N2, leads to symmetrization of the pharyngeal morphology.

These experiments are congruent with (i) the initial observation of asymmetrical expression of $N k x 2.1$, FoxE4, and Hand at N3 and (ii) the symmetrization of their expression patterns upon treatment with Nodal inhibitors (Figure 4N,O,P). Together, these data suggest that morphological asymmetry in amphioxus is specified during the neurula stages through asymmetrical expression of downstream organ-specific target genes.

\section{Discussion}

In this study, we demonstrate that expression of the Nodal signaling pathway members in amphioxus resembles that of their vertebrate orthologs and that Nodal is necessary for the left-sided expression of other downstream genes and for left-sided morphogenesis. Inhibition of Nodal results in (i) downregulation of the left-sided genes and bilateral expression of the otherwise right-sided genes and (ii) symmetrization of the larval body. Based on our detailed expression survey and time course experiments, we propose a scheme for LR axis establishment in amphioxus (Figure 8) and discuss it in an evolutionary context below.

\section{The conservation of the role of Nodal signaling in left-right patterning in chordates}

In vertebrates, the LR organizer directs the initial break of symmetry by induction of asymmetric gene expression in its vicinity. The asymmetry at the LR organizer is then transmitted to induce asymmetry in the lateral plate mesoderm, where induction of asymmetric gene expression is followed by self-enhancement and amplification of the signals that result in polarization of the body, and establishment of left and right identities. Finally, these signals target organ-specific factors that are responsible for tissue-specific morphogenesis and differentiation of left- and right-sided organs. Our study shows that the Nodal signaling pathway patterns the LR axis and specifies the left side during early amphioxus embryogenesis principally in a similar way to that observed in vertebrates (Figure 8). In amphioxus, however, Nodal acts at the place of its initial asymmetric induction, and thus, there is no transfer of asymmetrically induced signals from the organizer to the lateral plate mesoderm.

We showed that Nodal and its co-ligand Gdf1/3 are expressed in a bilateral fashion at the onset of neurulation (N0) and become confined to the left side during subsequent stages, whereas Nodal inhibitors Cerberus and Lefty display early asymmetric expression. Previous studies showed that Cerberus expression changes from bilateral to unilateral at the gastrula/neurula transition [37]. This change resembles that of its orthologs, Cerl 2 in the mouse and Coco in Xenopus, during specification of bilateral asymmetry at the LR organizer [71-73]. The organizer houses monociliated cells, the cilia of which beat in a simultaneous manner to generate a leftward fluid flow. The flow promotes decay of Cerl 2 mRNA on the left side of the node [74], and a similar mechanism may also affect Coco in Xenopus [73]. On the left side, lack of inhibition by Cerl 2 results in local activation of Nodal that further suppresses expression of Cerl2, whereas on the right side, expression of Cerl2 inhibits action of Nodal [75]. Our data suggest that suppression of Cerberus by Nodal may also take place in amphioxus, given that inhibition of Nodal at the neurula stages results in ectopic activation of Cerberus expression on the left side (Figure 4D,I). Thus, although amphioxus Nodal and $G d f 1 / 3$ are expressed symmetrically at N0, their right-sided suppression by Cerberus results in propagation of the Nodal signal on the left side.

Simultaneous right-sided inhibition and left-sided activation of Nodal causes a bias towards left-sided expression of the members of this pathway at the N0 to N1 transition. In vertebrates, asymmetric activity at the LR organizer is translated into asymmetry in the lateral plate mesoderm $[10-12,76,77]$. Here, members of the Nodal pathway constitute a 'self-enhancement and lateral inhibition (SELI) system' that reinforces determination of the left side [13]. Nodal activates its own expression and thus establishes the identity of the left side, whereas its inhibitor Lefty 2 restricts Nodal activity to the left lateral plate mesoderm and prevents ectopic activation of this pathway on the right side [78]. Although no transfer of the LR signal occurs in amphioxus, the left-sided activation of the Nodal pathway at N1 is comparable to the vertebrate LR SELI system. In amphioxus, such 'self-enhancement' would be driven by Nodal, the inhibition of which results in the loss of expression of Nodal, Gdf1/3, Lefty, and Pitx, and leads to the loss of left-sided larval morphology. On the other hand, the 'lateral inhibition' would be provided by Lefty; the broad left-sided expression of this factor, which spans up to the embryonic midline, is reminiscent of the Nodalregulating, left-sided expression of Lefty 2 and the midline expression of Lefty1 in the mouse [79-81]. After propagation of the SELI system on the left side in amphioxus, 


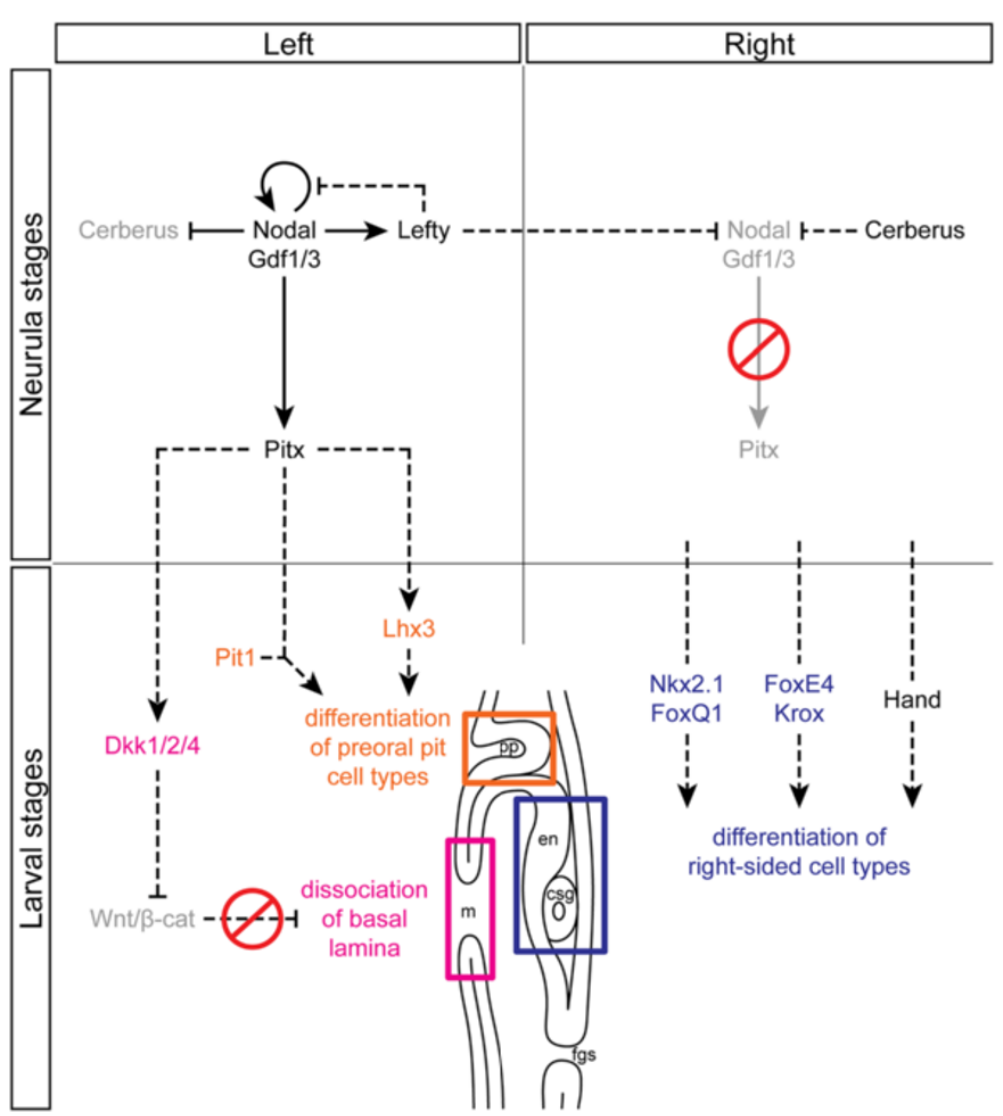

Figure 8 Model of the Nodal signaling pathway during establishment of the LR asymmetry in amphioxus. Dashed lines mark proposed interactions based on the data from other chordates. Gray text denotes inhibition of expression of the respective factors. During neurulation, Nodal and its co-ligand Gdf1/3 are expressed symmetrically. However, right-sided inhibition of Nodal by Cerberus results in propagation of the pathway on the left side, where Nodal activates expression of Nodal, Gdf1/3, Lefty, and Pitx and inhibits expression of Cerberus. We propose that during the course of later development, Pitx interacts with organ-specific factors and promotes left-side specific morphogenesis. Anteriorly, Pitx is proposed to interact with Pit 1 and to activate expression of $L h x 3$ to promote differentiation of cell types within the preoral pit. Slightly more posterior, Pitx is proposed to regulate expression of $D k k 1 / 2 / 4$, which inhibits Wnt/ $\beta$-catenin signaling, and thus promotes fusion of ectoderm and endoderm epithelia during morphogenesis of the mouth opening. The mechanism underlying activation of expression of factors responsible for right-sided morphogenesis is currently unknown. csg, club-shaped gland; en, endostyle; fgs, first gill slit; m, mouth; pp, preoral pit.

the midline expression of Cerberus at N2 can further block the potential transfer and ectopic activation of Nodal signaling on the right side (Figure 2O). Our time course experiments show that normal amphioxus larval morphology is susceptible to Nodal inhibition, especially at N0 to N2, and we propose that the LR SELI system is active during this period.

The first signs of morphological asymmetry in amphioxus are observed after the LR SELI stages at N2 to N3, in which the embryos exhibit asymmetrical arrangement of somites and a unilaterally biased expression of organ-specific genes. In vertebrates, the LR morphological asymmetry is conveyed by Pitx2 [82-87]. It is well understood that organs individually reply to the asymmetric Pitx 2 signal, via differential proliferation, differentiation, cell shape changes, or apoptosis, but the molecular mechanisms that link Pitx2 to organ-specific morphogenesis are largely unknown and are only just being deciphered (see [14] and citations therein). In amphioxus, the Nodal target Pitx probably also activates transcription of downstream factors in an organspecific manner, thus triggering the offset placement of left-sided somites and peripheral nerves as opposed to their right-sided counterparts and promoting development of the preoral pit, mouth, and duct of the clubshaped gland (discussed later).

\section{The significance of amphioxus left-right asymmetry} and its implications for the development and evolution of the chordate characteristics

The discovery that the Nodal pathway acts to promote asymmetric development in amphioxus is in a good 
accord with the situation in other chordates and even non-chordate deuterostomes and other bilaterians. However, it is challenging to relate the extreme asymmetrical morphology in amphioxus to the almost perfect external symmetry in tunicates and vertebrates. Recent molecular studies support the hypothesis that a dorso-ventral inversion may have occurred in the common ancestor of chordates (reviewed in $[25,26]$ ), although the exact mechanism leading to this inversion is still debated $[23,88-90]$. Amphioxus resides within the chordate clade that split off from the lineage leading to tunicates and vertebrates just after this event. Therefore, it is difficult to determine whether the profound amphioxus asymmetry is somehow related to this event, that is, whether it reflects a recapitulation of the past evolutionary history, and thus represents a primitive chordate condition, or whether it exemplifies a peculiarity of the cephalochordate lineage with no relation to tunicates or vertebrates. The mouth plays a pivotal role in this discussion, as its presence on the left side in amphioxus larvae casts doubts on its supposed homology with the median mouth of other chordates [91].

In tunicates and vertebrates, the mouth develops at the anterior neural boundary [92,93]. This boundary, referred to as the preplacodal ectoderm, is the location at which sensory placodes, cement organs, and the mouth originate and is demarcated by expression of members of the
Pax, Six, Eya, and Dach families of transcription factors [94]. Pitx factors define the anterior-most domain within the preplacodal ectoderm and are responsible for development of the mouth, cement organs, adenohypophysis, and lens [95]. Pitx factors are downstream of bone morphogenetic protein (BMP) signaling (an epidermal cue) and Otx2 (a neural cue) [96,97]. This initial anterior expression of Pitx factors is distinct from later left-sided Pitx2 expression that acts downstream of Nodal signaling and is responsible for the LR asymmetry. Additionally, both the anterior and left-sided expression of Pitx factors is regulated at the transcriptional level by distinct BMP (Smad1/5)- and Nodal (FoxH1)-responsive elements [87,98,99].

In non-chordate deuterostomes, the mouth seems to be specified and regulated differently from that of vertebrates (Figure 9). In echinoderms, opposing Nodal/BMP signaling organizes the oral-aboral (dorso-ventral) axis, with Nodal signaling promoting, but BMP signaling inhibiting, the oral fate [100-102]. Interestingly, Pitx2 does not seem to be part of the gene regulatory network promoting oral fate in echinoderms; if expression is observed in the oral ectoderm of an echinoderm species, it occurs only at later stages and in relation to LR asymmetry [103]. In hemichordates, the mouth develops on the non-Bmp side of the embryonic body (akin to mouth

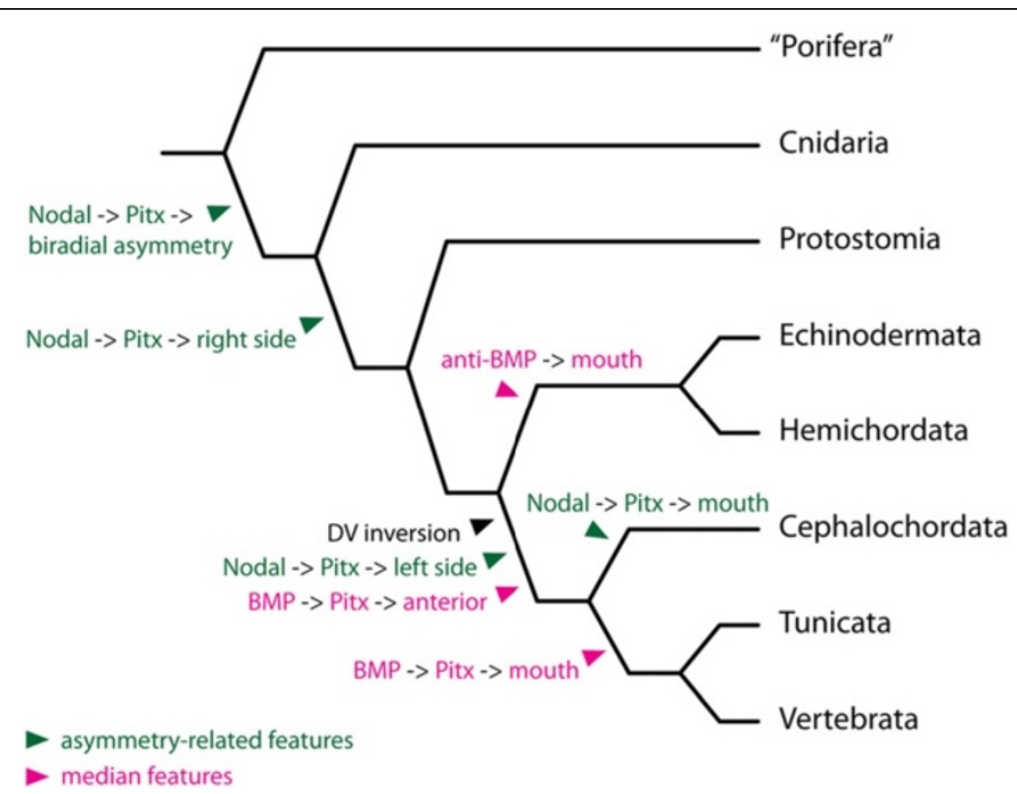

Figure 9 Hypothetical scenario of the role of Pitx in LR asymmetry and mouth development across metazoans. Green text marks LR asymmetric expression; magenta text marks median expression. In protostomes, echinoderms and hemichordates, the Nodal-Pitx pathway regulates determination of the right side. In cephalochordates, tunicates and vertebrates, on the other hand, this pathway determines the left side due to inversion of the body axes, an event that supposedly occurred at the base of chordate phylogeny. Concomitantly, new Pitx expression downstream of BMP signaling occurred at the anterior neural boundary (this interaction remains to be confirmed in amphioxus). Mouth development seems to be regulated differently between different deuterostome lineages. BMP signaling suppresses oral development in echinoderms and hemichordates, while it promotes oral development through Pitx in tunicates and vertebrates. In the cephalochordate amphioxus, the Nodal-Pitx pathway regulates LR asymmetry and is also responsible for development of the mouth, whose relation to the mouth of other chordates is contested. 
development in echinoderms), while Pitx expression can be found on the opposite side in the region of the prospective proboscis pore [104]. This structure has traditionally been proposed to be homologous to the anterior pituitary of vertebrates and the preoral pit of amphioxus [105]. The regulation of Pitx downstream of BMP signaling in hemichordates [104] is reminiscent of the situation in tunicates and vertebrates and has been proposed as a key factor of the scenario for evolutionary repositioning of the mouth. According to this scenario, the position of the mouth in the chordate ancestor changed from echinoderm- and hemichordate-like anti-BMP and nonPitx territory to tunicate- and vertebrate-like pro-BMP and Pitx-expressing territory [92].

Amphioxus occupies a key position in this scenario and was previously proposed to exhibit a situation similar to that of vertebrates [92]. Yet, a bona fide vertebrate-like preplacodal ectoderm is not present at the anterior neural boundary $[94,106]$, and members of the Six, Eya, Pax, and Dach families are expressed elsewhere throughout the body [107]. Yet, Pitx seems to be transiently expressed at the anterior neural boundary at the late gastrula stage (G6), and this expression coincides with the partially overlapping bilateral expression of Nodal [39,40]. However, this anterior Pitx expression is diminished at the early pre-hatching neurula stage (N0), and a new left-sided domain of Pitx expression is induced at the N0 to N1 transition (Figure 2E,F). Additionally, while treatments with Nodal inhibitors at neurula stages result in loss of the mouth, treatments spanning the late gastrula stages have only minor effects on mouth development (Figure 7). This suggests that either Pitx expression is not triggered by the Nodal signaling at these early stages or that it is not necessary for the development of the mouth. Currently, it is unknown whether the anterior Pitx expression is downstream of neural Otx and epidermal BMP signaling and whether this circuitry plays any role in the development of the mouth, as in other chordates. Future research should therefore be aimed at uncovering the role of anterior Pitx expression in amphioxus, to determine whether this feature represents a shared characteristic between tunicates and vertebrates [108] or has a deeper phylogenetic significance.

The peculiar left-sided mouth in amphioxus has long been a matter of debate as to whether or not it is homologous to the median mouth of vertebrates. Van Wijhe [109-111] argued that the amphioxus mouth is actually a modified first gill slit, which corresponds to the left spiracle of vertebrates. The presence of this left-sided opening would be advantageous for filter feeding during counter-clockwise rotation of the amphioxus larva; the original median mouth may have become greatly diminished, with its remnants present in the preoral pit. This rather controversial assumption has been contested by those who propose affiliation of the left-sided mouth of amphioxus with that of vertebrates. MacBride [112] suggested that the pharyngeal structures in amphioxus, including the mouth, are initiated in a primarily symmetrical manner and that larval pharyngeal asymmetry can be explained by differential growth within the pharynx and the resultant displacement of organs. Willey [113], on the other hand, proposed that the development of the asymmetrical pharynx in amphioxus with its left-sided mouth could be explained as an evolutionary consequence of the rostral prolongation of notochord. This prolongation would cause the pharynx to undergo a counter-clockwise torsion (when seen from behind) that would bring the anlagen of dorsal organs, like the mouth and preoral pit (see [114]), to the left side and those of ventral organs, like the endostyle and the club-shaped gland, to the right side $[35,88,113]$. Such morphogenesis could potentially be explained by differential growth or by cellular rearrangements of surrounding tissues, as proposed by MacBride [112], under the control of Nodal signaling. To date, cellular behavior during morphogenesis of the amphioxus pharynx has not been studied in depth. Holland and Holland [115] reported that cell proliferation is not correlated with potential pharyngeal torsion; rather, it is the displaced organs themselves, and not the surrounding tissues, that exhibit increased proliferation. However, if we propose that the pharyngeal organs are primarily established symmetrically and that the Nodal pathway controls the torsion, the anlagen of individual organs should remain at their supposed median position upon inhibition of this pathway (that is, the mouth and the preoral pit would be positioned dorsally and the endostyle and the club-shaped gland ventrally). In contrast, our results show that inhibition of the Nodal pathway results in the duplication of right-sided structures and does not result in the supposed development of medially positioned mouth or endostyle (Figure 6). This implies that the primordia of the pharyngeal structures are established primarily asymmetrically and that their asymmetrical arrangement is probably not a secondary consequence of the torsion.

Another option is that the mouth in amphioxus is a derived cephalochordate characteristic with no counterpart in other chordates. Yasui and Kaji [91] argued that structures associated with the mouth during amphioxus metamorphosis as well as those associated with the mouth of the larval lamprey represent traits obtained independently due to similar feeding habits and thus demonstrate analogically derived characteristics. This hypothesis, together with the findings that mouth development is regulated by medially expressed Pitx (downstream of BMP signaling) in tunicates and vertebrates and the sinistrally expressed Pitx (downstream of 
Nodal signaling) in amphioxus, raises serious questions regarding the a priori proposition of the homology of oral openings across chordates (Figure 9).

Despite the contested relationships among chordate mouths, the left-sided Pitx expression in amphioxus may serve similar functions as its vertebrate orthologs expressed medially at the anterior neural boundary. For example, mouse Pitx1 and Pitx2 regulate both early morphogenesis of Rathke's pouch and later proliferation of pituitary cell precursors [116,117]. Pitx1 activates expression of $L h \times 3$ and acts in synergy with POU1F1/Pit1 to promote differentiation of pituitary cell types [118]. Both $L h \times 3$ and Pit 1 homologs are expressed in the amphioxus preoral pit $[52,119]$, and thus, it is possible that they interact with upstream Pitx (Figure 8). Similarly, Pitx can regulate expression of $D k k 1 / 2 / 4$ in the amphioxus mouth and preoral pit. Previously, it was shown that $D k k 1$ is co-expressed with Pitx factors in the oral region of Xenopus [120,121]. Moreover, microarray analysis demonstrated that activators of the $\mathrm{Wnt} / \beta$-catenin pathway are downregulated, while inhibitors of this pathway, including $D k k 1$, become upregulated, in the frog oral region; additionally, $D k k 1$ overexpression results in an enlarged mouth [122]. Local inhibition of the Wnt/B-catenin pathway in the mouth region is then necessary for proper dissolution of the basal lamina between the ectoderm and the endoderm and for the subsequent break of the oral membrane. A direct link between Pitx factors and Dkk1 during vertebrate mouth development has not been identified at the time of writing, but Pitx2 has been shown to activate expression of $D k k 2$ during development of the anterior segment of the eye in mouse [123]. The proposed regulation of $D k k 1 / 2 / 4$ expression by Pitx and local inhibition of $\mathrm{Wnt} / \beta$-catenin signaling by Dkk1/2/4 may thus together form an ancient mechanism to promote fusion between ectoderm and endoderm epithelia, as a prerequisite for normal morphogenesis of the amphioxus mouth and preoral pit.

We have shown here that Nodal signaling is responsible for establishment of the bilateral asymmetry of amphioxus paraxial structures, reminiscent of the role of Nodal in LR asymmetry on the pharyngeal region. The break of the initial symmetrical arrangement of paraxial structures starts at the mid-neurula stage (N2), when the fifth left somite displays a slightly anterior position and advanced differentiation to its right counterpart [124]. Shortly after (at early N3), this somite asymmetry is followed by asymmetrical development of axons of peripheral nerves [125]. During the course of development, the asymmetrical arrangement of somites and peripheral nerves is amplified, and new somites arise in a primarily asymmetric manner. Both Nodal and Pitx are expressed in the left anterior archenteron during outpocketing of the first somites (this study and $[39,40]$ ). We have shown that inhibition of Nodal signaling results in (i) the symmetrical arrangement of somites by the late neurula stage (N3) and (ii) consequent development of bilaterally symmetrical muscle segments and peripheral nerves, resulting in the left side becoming aligned with the right side. The offset arrangement of somites regulated by Nodal signaling is specific to amphioxus development; however, the asymmetrical arrangement itself may also represent a basis for vertebrate somitogenesis. Previous experiments showed that depletion of retinoic acid in the zebrafish, chick, or mouse causes temporal acceleration of somite development on the left side [126-128], a situation reminiscent of normal development in amphioxus. Blum et al. [1] therefore proposed that retinoic acid signaling may act to shield vertebrate somites from Nodal signaling, which, in turn, may explain the vertebrate-specific transfer of the Nodal signal from the LR organizer to the lateral plate mesoderm without affecting the somites. Retinoic acid signaling thus seems to be important for buffering the lateralizing effects on vertebrate somitogenesis, while the LR alternation of somites might be a primitive feature of chordate somitogenesis.

\section{Symmetry breaking in amphioxus: hypotheses and prospects}

A further area of interest regarding LR asymmetry establishment is how the symmetry is initially broken in amphioxus. In the majority of vertebrates, symmetry breaking is associated with cilia in the LR organizer, which drive the fluid flow of certain factors to the left and thereby induce bilaterally symmetrical gene expression $[5,129]$. On the other hand, there are reports that uneven distribution of ion channels during early cleavage may cause differences in $\mathrm{pH}$ and membrane potential between the left and right sides of embryos of certain vertebrate species, resulting in symmetry breaking [7]. Blum et al. [1] proposed that the cilia-driven flow may actually be an ancient feature dating back to the common ancestor of deuterostomes. Indeed, the dorsal wall of the archenteron of amphioxus embryo possesses monociliated cells $[58,59]$, and this region is reminiscent of the LR organizer in Xenopus [1]. Furthermore, it is worth noting that ciliogenesis occurs during the gastrula stage in amphioxus, and cilia at the outside epidermis likely start to function at very late gastrula stage (G6 to G7) to enable embryo rotation within the fertilization membrane from this stage onward [58]. However, it is unclear whether the monocilia in the dorsal wall of archenteron can also move and generate fluid flow [2]; future live-imaging studies are needed to answer this question. Nevertheless, ciliogenesis and embryonic rotation appear to commence just prior to the onset of asymmetric gene expression of Cerberus, Lefty, and Nodal (Figure 2), suggesting that cilia movement may play some roles in symmetry breaking in amphioxus. This hypothesis could be 
tested by examining the effects of blocking ciliogenesis or cilia movement on LR asymmetric development in amphioxus embryos.

It is also unclear whether uneven distribution of ion channels influences symmetry breaking in amphioxus. Although there is clear evidence for uneven distribution of maternal transcripts of germline-related genes between the first two blastomeres of the amphioxus embryo, these maternal transcripts can be deposited into either the left or the right blastomere after the first cleavage [55,60]. It is unknown whether other maternal or early zygotic transcripts exhibit asymmetric distribution across the LR axis in cleavage-stage amphioxus embryos. Further surveys on early mRNA/protein distribution and pharmacological block of specific ion channels in cleavage-stage embryos are required to determine whether ion flux is involved in the symmetry breaking in amphioxus. We anticipate that the results obtained from amphioxus will provide important information for understanding the evolution of LR patterning mechanisms in chordates.

\section{Conclusions}

We have shown that Nodal signaling is necessary for the establishment of LR asymmetry and for the determination of the left side of the amphioxus body. Our expression analysis and time course experiments on carefully staged neurulae allowed us to follow critical steps during the establishment of LR asymmetry; moreover, our experimental design provides a basis for future research into the existence of leftward flow-generating cilia in amphioxus and their putative role in initial symmetry breaking [1]. Given the phylogenetic position of amphioxus, such studies promise to cast light on the shared and derived character states of LR asymmetry establishment among chordates.

\section{Additional files}

Additional file 1: Table S1. List of clones from the EST library used to synthesize probes of $B$. floridae genes.

Additional file 2: Table S2. List of PCR primers used for amplifying CDNA fragments of $B$. floridae and B. lanceolatum genes.

Additional file 3: Figure S1. Branchiostoma floridae larvae display similar morphological changes upon treatment with SB505124. Asterisks (*) mark the anterior, ' $L$ ' marks the left side, and ' $R$ ' marks the right side. Scale bar, $25 \mu \mathrm{m}$. (A, A', B, B') Pitx expression marks the left-sided mouth (black arrow) and preoral pit (black arrowhead); both structures are lost upon treatment with SB505124. (C, C', D, D') FoxE4 expression marks the whole club-shaped gland (white arrow), which resides mainly on the right side of the larva. Upon treatment, the club-shaped gland appears symmetrically on both the left and right sides. (E, E', F, F') Nkx2.1 is expressed in the right-sided endostyle (white arrowhead). In the embryos treated with SB505124, the endostyle forms on both the left and right sides.

Additional file 4: Video S1. A 3D model reconstructed from z-stack images of pharyngeal structures in a control $B$. floridae larva. The anterior is pointed towards the left side. The initial view of the larva is a lateral view, which slightly tilts towards the left side. The larva is then rotated clockwise when viewed from the posterior end. The preoral pit (green) opens on the left side, but the organ itself resides up to the midline. The position of the left-sided mouth has been indicated by highlighting the cells around it (yellow). The endostyle (blue) resides on the right side, anterior to the club-shaped gland (red); the dorsal part of the club-shaped gland is situated mainly on the right side, while the ventral part crosses over to the left. The first two gill slits (purple) reside behind the endostyle. The first gill slit appears tilted towards the right, and the opening emerges on the right side; the second gill slit forms in a more medial position.

Additional file 5: Video S2. A 3D model reconstructed from z-stack images of pharyngeal structures in a B. floridae larva treated with SB505124. The anterior is pointed towards the left side. The initial view of the larva is a lateral view, which tilts slightly towards the left side. The larva is rotated clockwise when viewed from the posterior end. The preoral pit does not form in the treated larva, leaving a vacant region within the rostrum. The mouth does not open in the treated larva. The endostyle (blue) forms ectopically on the left side, resulting in a ' $U$ '-shaped organ. The club-shaped gland (red), still posterior to the endostyle, also forms a ' $U$ '-shaped structure, caused by the ectopic formation of the organ on the left. The first two gill slits (purple) are both located medially in the treated larva.

\section{Abbreviations}

BLAST: Basic Logical Alignment Search Tool; BMP: bone morphogenetic protein; CNS: central nervous system; DIG: digoxigenin; DMSO: dimethyl sulfoxide; EST: expressed sequence tag; LR: left-right; SELI: self-enhancement and lateral inhibition; tBLASTx: translated nucleotide BLAST; TGF- $\beta$ : transforming growth factor beta.

\section{Competing interests}

The authors declare that they have no competing interests.

\section{Authors' contributions}

VS, ZK, and JKY conceived and designed the experiments. VS, LWY, TML, SWH, and JKY performed the experiments. VS, LWY, TML, and JKY analyzed the data. VS, LWY, and JKY wrote the paper. All authors read and approved the final manuscript.

\section{Acknowledgements}

We thank Linda Holland and Nicholas Holland at the Scripps Institution of Oceanography, UCSD, and Daniel Meulemans Medeiros at the University of Colorado, Boulder, for collecting the $B$. floridae adults. We are grateful to Hector Escriva and Stephanie Bertrand at Laboratoire Arago, Banyuls-sur-Mer, France, for providing the B. lanceolatum adults and the B.I. Krox clone. We also thank Cho-Fat Hui, Director of the ICOB Marine Research Station, and Che-Huang Tung, Meng-Yun Tang, Tzu-Kai Huang, Veronika Noskova, and Jindra Pohorela for culturing the amphioxus in our laboratories. We thank Dr. Shao-Chun Hsu in the ICOB core facility for the technical support with confocal microscopy. We thank Sarka Takacova and Dr. Duncan Wright for the English editing. We also thank the three anonymous reviewers for their helpful comments. VS is supported by the Grant Agency of the Czech Republic (14-20839P). The amphioxus work in the lab of ZK is supported in part by grant no. LH12047 from the Ministry of Education, Youth and Sports of the Czech Republic and by IMG institutional support RVO68378050. JKY is supported by the Ministry of Science and Technology, Taiwan (101-2923-B-001-004-MY2; 102-2311-B-001011-MY3) and by a Career Development Award from Academia Sinica, Taiwan (AS-98-CDA-L06).

\section{Author details}

${ }^{1}$ Institute of Molecular Genetics, Academy of Sciences of the Czech Republic, Videnska 1083, Prague 14220, Czech Republic. Institute of Cellular and Organismic Biology, Academia Sinica, 128 Academia Road, Section 2, Nankang, Taipei 11529, Taiwan. ${ }^{3}$ Institute of Oceanography, National Taiwan University, 1 Roosevelt Road, Section 4, Taipei 10617, Taiwan.

Received: 17 November 2014 Accepted: 27 January 2015

Published: 17 February 2015 


\section{References}

1. Blum M, Feistel K, Thumberger T, Schweickert A. The evolution and conservation of left-right patterning mechanisms. Development. 2014;141(8):1603-13.

2. Namigai EK, Kenny NJ, Shimeld SM. Right across the tree of life: the evolution of left-right asymmetry in the Bilateria. Genesis. 2014;52(6):458-70.

3. Palmer AR. Animal asymmetry. Curr Biol. 2009;19(12):R473-7.

4. Raya A, Izpisua Belmonte JC. Left-right asymmetry in the vertebrate embryo: from early information to higher-level integration. Nat Rev Genet. 2006;7(4):283-93.

5. Blum M, Schweickert A, Vick P, Wright CV, Danilchik MV. Symmetry breakage in the vertebrate embryo: when does it happen and how does it work? Dev Biol. 2014;393(1):109-23.

6. Shiratori $\mathrm{H}$, Hamada $\mathrm{H}$. The left-right axis in the mouse: from origin to morphology. Development. 2006;133(11):2095-104.

7. Vandenberg LN, Levin M. A unified model for left-right asymmetry? Comparison and synthesis of molecular models of embryonic laterality. Dev Biol. 2013:379(1):1-15.

8. Su YH. Telling left from right: left-right asymmetric controls in sea urchins. Genesis. 2014;52(3):269-78.

9. Tanaka C, Sakuma R, Nakamura T, Hamada H, Saijoh Y. Long-range action of Nodal requires interaction with GDF1. Genes Dev. 2007;21(24):3272-82.

10. Brennan J, Norris DP, Robertson EJ. Nodal activity in the node governs leftright asymmetry. Genes Dev. 2002;16(18):2339-44.

11. Kawasumi A, Nakamura T, Iwai N, Yashiro K, Saijoh Y, Belo JA, et al. Left-right asymmetry in the level of active Nodal protein produced in the node is translated into left-right asymmetry in the lateral plate of mouse embryos. Dev Biol. 2011;353(2):321-30.

12. Saijoh $Y$, Oki S, Ohishi S, Hamada H. Left-right patterning of the mouse lateral plate requires nodal produced in the node. Dev Biol. 2003;256(1):160-72.

13. Nakamura T, Mine N, Nakaguchi E, Mochizuki A, Yamamoto M, Yashiro K, et al. Generation of robust left-right asymmetry in the mouse embryo requires a self-enhancement and lateral-inhibition system. Dev Cell. 2006;11(4):495-504.

14. Nakamura T, Hamada H. Left-right patterning: conserved and divergent mechanisms. Development. 2012;139(18):3257-62.

15. Duboc V, Rottinger E, Lapraz F, Besnardeau L, Lepage T. Left-right asymmetry in the sea urchin embryo is regulated by nodal signaling on the right side. Dev Cell. 2005;9(1):147-58.

16. Luo YJ, Su YH. Opposing nodal and BMP signals regulate left-right asymmetry in the sea urchin larva. PLoS Biol. 2012;10(10):e1001402.

17. Morokuma J, Ueno M, Kawanishi H, Saiga H, Nishida H. HrNodal, the ascidian nodal-related gene, is expressed in the left side of the epidermis, and lies upstream of HrPitx. Dev Genes Evol. 2002;212(9):439-46.

18. Yoshida K, Saiga H. Left-right asymmetric expression of Pitx is regulated by the asymmetric Nodal signaling through an intronic enhancer in Ciona intestinalis. Dev Genes Evol. 2008;218(7):353-60.

19. Burdine RD, Caspary T. Left-right asymmetry: lessons from Cancun. Development. 2013;140(22):4465-70.

20. Grande C, Patel NH. Nodal signalling is involved in left-right asymmetry in snails. Nature. 2009;457(7232):1007-11.

21. Kuroda R, Endo B, Abe M, Shimizu M. Chiral blastomere arrangement dictates zygotic left-right asymmetry pathway in snails. Nature. 2009;462(7274):790-4.

22. Watanabe H, Schmidt HA, Kuhn A, Hoger SK, Kocagoz Y, Laumann-Lipp N, et al. Nodal signalling determines biradial asymmetry in Hydra. Nature. 2014;515(7525):112-5.

23. Arendt D, Nubler-Jung K. Inversion of dorsoventral axis? Nature. 1994;371(6492):26.

24. De Robertis EM, Sasai Y. A common plan for dorsoventral patterning in Bilateria. Nature. 1996;380(6569):37-40.

25. Holland LZ, Carvalho JE, Escriva H, Laudet V, Schubert M, Shimeld SM, et al Evolution of bilaterian central nervous systems: a single origin? EvoDevo. 2013;4(1):27.

26. De Robertis EM. Evo-devo: variations on ancestral themes. Cell. 2008;132(2):185-95.

27. Holland LZ, Albalat R, Azumi K, Benito-Gutierrez E, Blow MJ, Bronner-Fraser $M$, et al. The amphioxus genome illuminates vertebrate origins and cephalochordate biology. Genome Res. 2008;18(7):1100-11.

28. Putnam NH, Butts T, Ferrier DE, Furlong RF, Hellsten U, Kawashima T, et al. The amphioxus genome and the evolution of the chordate karyotype. Nature. 2008;453(7198):1064-71.
29. Bourlat SJ, Juliusdottir T, Lowe CJ, Freeman R, Aronowicz J, Kirschner M, et al. Deuterostome phylogeny reveals monophyletic chordates and the new phylum Xenoturbellida. Nature. 2006;444(7115):85-8.

30. Delsuc F, Tsagkogeorga G, Lartillot N, Philippe H. Additional molecular support for the new chordate phylogeny. Genesis. 2008;46(11):592-604.

31. Bertrand S, Escriva H. Evolutionary crossroads in developmental biology: amphioxus. Development. 2011;138(22):4819-30.

32. Schubert M, Holland LZ, Stokes MD, Holland ND. Three amphioxus Wnt genes (AmphiWnt3, AmphiWnt5, and AmphiWnt6) associated with the tail bud: the evolution of somitogenesis in chordates. Dev Biol. 2001;240(1):262-73.

33. Lu TM, Luo YJ, Yu JK. BMP and Delta/Notch signaling control the development of amphioxus epidermal sensory neurons: insights into the evolution of the peripheral sensory system. Development. 2012;139 (11):2020-30.

34. Glardon S, Holland LZ, Gehring WJ, Holland ND. Isolation and developmental expression of the amphioxus Pax-6 gene (AmphiPax-6): insights into eye and photoreceptor evolution. Development. 1998;125(14):2701-10.

35. Lacalli T. Mucus secretion and transport in amphioxus larvae: organization and ultrastructure of the food trapping system, and implications for head evolution. Acta Zool. 2008;89(3):219-30.

36. Boorman CJ, Shimeld SM. Pitx homeobox genes in Ciona and amphioxus show left-right asymmetry is a conserved chordate character and define the ascidian adenohypophysis. Evol Dev. 2002;4(5):354-65.

37. Le Petillon Y, Oulion S, Escande ML, Escriva H, Bertrand S. Identification and expression analysis of BMP signaling inhibitors genes of the DAN family in amphioxus. Gene Expr Patt. 2013;13(8):377-83.

38. Onai T, YU JK, Blitz IL, Cho KW, Holland LZ. Opposing Nodal/Ng1 and BMP signals mediate axial patterning in embryos of the basal chordate amphioxus. Dev Biol. 2010;344(1):377-89.

39. Yasui $K$, Zhang S, Uemura M, Saiga H. Left-right asymmetric expression of BbPtx, a Ptx-related gene, in a lancelet species and the developmental leftsidedness in deuterostomes. Development. 2000;127(1):187-95.

40. Yu JK, Holland LZ, Holland ND. An amphioxus nodal gene (AmphiNodal) with early symmetrical expression in the organizer and mesoderm and later asymmetrical expression associated with left-right axis formation. Evol Dev. 2002:4(6):418-25.

41. Yu JK, Satou Y, Holland ND, Shin IT, Kohara Y, Satoh N, et al. Axial patterning in cephalochordates and the evolution of the organizer. Nature. 2007:445(7128):613-7.

42. Venkatesh TV, Holland ND, Holland LZ, Su MT, Bodmer R. Sequence and developmental expression of amphioxus AmphiNk2-1: insights into the evolutionary origin of the vertebrate thyroid gland and forebrain. Dev Genes Evol. 1999;209(4):254-9.

43. Onimaru K, Shoguchi E, Kuratani S, Tanaka M. Development and evolution of the lateral plate mesoderm: comparative analysis of amphioxus and lamprey with implications for the acquisition of paired fins. Dev Biol. 2011;359(1):124-36.

44. Kusakabe R, Kusakabe T, Satoh N, Holland ND, Holland LZ. Differential gene expression and intracellular mRNA localization of amphioxus actin isoforms throughout development: Implications for conserved mechanisms of chordate development. Dev Genes Evol. 1997;207(4):203-15.

45. Satoh G, Wang Y, Zhang P, Satoh N. Early development of amphioxus nervous system with special reference to segmental cell organization and putative sensory cell precursors: a study based on the expression of panneuronal marker gene Hu/elav. J Exp Zool. 2001;291(4):354-64.

46. Yu JK, Wang MC, Shin IT, Kohara Y, Holland LZ, Satoh N, et al. A cDNA resource for the cephalochordate amphioxus Branchiostoma floridae. Dev Genes Evol. 2008;218(11-12):723-7.

47. Yu JK, Holland LZ, Jamrich M, Blitz IL, Hollan ND. AmphiFoxE4, an amphioxus winged helix/forkhead gene encoding a protein closely related to vertebrate thyroid transcription factor-2: expression during pharyngeal development. Evol Dev. 2002;4(1):9-15.

48. Langeland JA, Tomsa JM, Jackman Jr WR, Kimmel CB. An amphioxus snail gene: expression in paraxial mesoderm and neural plate suggests a conserved role in patterning the chordate embryo. Dev Genes Evol. 1998;208(10):569-77.

49. Mazet F, Luke GN, Shimeld SM. The amphioxus FoxQ1 gene is expressed in the developing endostyle. Gene Expr Patt. 2005;5(3):313-5.

50. Ogasawara M. Overlapping expression of amphioxus homologs of the thyroid transcription factor-1 gene and thyroid peroxidase gene in the endostyle: insight into evolution of the thyroid gland. Dev Genes Evol. 2000;210(5):231-42. 
51. Somorjai I, Bertrand S, Camasses A, Haguenauer A, Escriva H. Evidence for stasis and not genetic piracy in developmental expression patterns of Branchiostoma lanceolatum and Branchiostoma floridae, two amphioxus species that have evolved independently over the course of $200 \mathrm{Myr}$. Dev Genes Evol. 2008;218(11-12):703-13.

52. Wang Y, Zhang PJ, Yasui K, Saiga H. Expression of Bblhx3, a LIM-homeobox gene, in the development of amphioxus Branchiostoma belcheri tsingtauense. Mech Dev. 2002;117(1-2):315-9.

53. Zhang $Y$, Mao B. Embryonic expression and evolutionary analysis of the amphioxus Dickkopf and Kremen family genes. J Genet Genomics. 2010;37(9):637-45.

54. Oulion S, Bertrand S, Belgacem MR, Le Petillon Y, Escriva H. Sequencing and analysis of the Mediterranean amphioxus (Branchiostoma lanceolatum) transcriptome. PLoS One. 2012;7(5):e36554.

55. Zhang QJ, Luo YJ, Wu HR, Chen YT, Yu JK. Expression of germline markers in three species of amphioxus supports a preformation mechanism of germ cell development in cephalochordates. EvoDevo. 2013;4(1):17.

56. Yu JK, Holland LZ. Amphioxus (Branchiostoma floridae) spawning and embryo collection. Cold Spring Harbor Protocols. 2009. doi:10.1101/pdb. prot5285.

57. Fuentes M, Benito E, Bertrand S, Paris M, Mignardot A, Godoy L, et al. Insights into spawning behavior and development of the European amphioxus (Branchiostoma lanceolatum). J Exp Zool B Mol Dev Evol. 2007;308(4):484-93.

58. Hirakow R, Kajita N. Electron microscopic study of the development of amphioxus, Branchiostoma belcheri tsingtauense: the gastrula. J Morphol. 1991;207:27-52.

59. Hirakow R, Kajita N. Electron microscopic study of the development of amphioxus, Branchiostoma belcheri tsingtauense: the neurula and larva. Acta Anat Nippon. 1994;69:1-13.

60. Wu HR, Chen YT, Su YH, Luo YJ, Holland LZ, Yu JK. Asymmetric localization of germline markers Vasa and Nanos during early development in the amphioxus Branchiostoma floridae. Dev Biol. 2011;353(1):147-59.

61. Holland LZ, Holland PWH, Holland ND. Revealing homologies between body parts of distantly related animals by in situ hybridization to developmental genes: amphioxus versus vertebrates. In: Ferraris JD, Palumbi SR, editors. Molecular zoology: advances, strategies, and protocols. New York: Wiley-Liss; 1996. p. 267-82. 473-483.

62. Hiruta J, Mazet F, Yasui K, Zhang P, Ogasawara M. Comparative expression analysis of transcription factor genes in the endostyle of invertebrate chordates. Dev Dyn. 2005;233(3):1031-7.

63. Lacalli TC. The dorsal compartment locomotory control system in amphioxus larvae. J Morphol. 2002;252(3):227-37.

64. Lacalli TC, Kelly SJ. Somatic motoneurons in amphioxus larvae: cell types, cell position and innervation patterns. Acta Zool. 1999;80:113-24.

65. Wicht H, Lacalli TC. The nervous system of amphioxus: structure, development, and evolutionary significance. Can J Zool. 2005;83(1):122-50.

66. Bardet PL, Schubert M, Horard B, Holland LZ, Laudet V, Holland ND, et al. Expression of estrogen-receptor related receptors in amphioxus and zebrafish: implications for the evolution of posterior brain segmentation at the invertebrate-to-vertebrate transition. Evol Dev. 2005;7(3):223-33.

67. Jackman WR, Langeland JA, Kimmel CB. islet reveals segmentation in the amphioxus hindbrain homolog. Dev Biol. 2000;220(1):16-26.

68. Li KL, Lu TM, YU JK. Genome-wide survey and expression analysis of the bHLH-PAS genes in the amphioxus Branchiostoma floridae reveal both conserved and diverged expression patterns between cephalochordates and vertebrates. EvoDevo. 2014:5:20.

69. Mazet F, Shimeld SM. The evolution of chordate neural segmentation. Dev Biol. 2002;251(2):258-70.

70. Jackman WR, Kimmel CB. Coincident iterated gene expression in the amphioxus neural tube. Evol Dev. 2002;4(5):366-74.

71. Inacio JM, Marques S, Nakamura T, Shinohara K, Meno C, Hamada H, et al. The dynamic right-to-left translocation of Cerl2 is involved in the regulation and termination of Nodal activity in the mouse node. PLoS One. 2013;8(3):e60406.

72. Marques S, Borges AC, Silva AC, Freitas S, Cordenonsi M, Belo JA. The activity of the Nodal antagonist Cerl-2 in the mouse node is required for correct L/R body axis. Genes Dev. 2004;18(19):2342-7.

73. Schweickert A, Vick P, Getwan M, Weber T, Schneider I, Eberhardt M, et al. The nodal inhibitor Coco is a critical target of leftward flow in Xenopus. Curr Biol. 2010;20(8):738-43.

74. Nakamura T, Saito D, Kawasumi A, Shinohara K, Asai Y, Takaoka K, et al. Fluid flow and interlinked feedback loops establish left-right asymmetric decay of Cerl2 mRNA. Nat Commun. 2012;3:1322.
75. Oki S, Kitajima K, Marques S, Belo JA, Yokoyama T, Hamada H, et al. Reversal of left-right asymmetry induced by aberrant Nodal signaling in the node of mouse embryos. Development. 2009;136(23):3917-25.

76. Pagan-Westphal SM, Tabin CJ. The transfer of left-right positional information during chick embryogenesis. Cell. 1998;93(1):25-35.

77. Vonica A, Brivanlou AH. The left-right axis is regulated by the interplay of Coco, Xnr1 and derriere in Xenopus embryos. Dev Biol. 2007;303(1):281-94.

78. Marjoram L, Wright C. Rapid differential transport of Nodal and Lefty on sulfated proteoglycan-rich extracellular matrix regulates left-right asymmetry in Xenopus. Development. 2011;138(3):475-85.

79. Meno C, Shimono A, Saijoh Y, Yashiro K, Mochida K, Ohishi S, et al. lefty-1 is required for left-right determination as a regulator of lefty-2 and nodal. Cell. 1998;94(3):287-97.

80. Meno C, Takeuchi J, Sakuma R, Koshiba-Takeuchi K, Ohishi S, Saijoh Y, et al. Diffusion of nodal signaling activity in the absence of the feedback inhibitor Lefty2. Dev Cell. 2001;1(1):127-38.

81. Yamamoto M, Mine N, Mochida K, Sakai Y, Saijoh Y, Meno C, et al. Nodal signaling induces the midline barrier by activating Nodal expression in the lateral plate. Development. 2003;130(9):1795-804.

82. Campione M, Steinbeisser H, Schweickert A, Deissler K, van Bebber F, Lowe LA, et al. The homeobox gene Pitxz: mediator of asymmetric left-right signaling in vertebrate heart and gut looping. Development. 1999;126(6):1225-34.

83. Kitamura K, Miura H, Miyagawa-Tomita S, Yanazawa M, Katoh-Fukui Y, Suzuki $\mathrm{R}$, et al. Mouse Pitx2 deficiency leads to anomalies of the ventral body wall, heart, extra- and periocular mesoderm and right pulmonary isomerism. Development. 1999;126(24):5749-58.

84. Lin CR, Kioussi C, O'Connell S, Briata P, Szeto D, Liu F, et al. Pitx2 regulates lung asymmetry, cardiac positioning and pituitary and tooth morphogenesis. Nature. 1999;401(6750):279-82.

85. Lu MF, Pressman C, Dyer R, Johnson RL, Martin JF. Function of Rieger syndrome gene in left-right asymmetry and craniofacial development. Nature. 1999;401(6750):276-8.

86. Ryan AK, Blumberg B, Rodriguez-Esteban C, Yonei-Tamura S, Tamura K, Tsukui T, et al. Pitx2 determines left-right asymmetry of internal organs in vertebrates. Nature. 1998;394(6693):545-51.

87. Shiratori H, Yashiro K, Shen MM, Hamada H. Conserved regulation and role of Pitx2 in situs-specific morphogenesis of visceral organs. Development. 2006;133(15):3015-25.

88. Lacalli TC. Head organization and the head/trunk relationship in protochordates: problems and prospects. Int Comp Biol. 2008;48(5):620-9.

89. Satoh N. An aboral-dorsalization hypothesis for chordate origin. Genesis. 2008;46(11):614-22.

90. Gerhart J, Lowe C, Kirschner M. Hemichordates and the origin of chordates. Curr Opin Gen Dev. 2005;15(4):461-7.

91. Yasui K, Kaji T. The lancelet and ammocoete mouths. Zool Sci. 2008;25(10):1012-9.

92. Christiaen L, Jaszczyszyn Y, Kerfant M, Kano S, Thermes V, Joly JS. Evolutionary modification of mouth position in deuterostomes. Semin Cell Dev Biol. 2007;18(4):502-11.

93. Soukup V, Horacek I, Cerny R. Development and evolution of the vertebrate primary mouth. J Anat. 2013;222(1):79-99.

94. Schlosser G. Evolutionary origins of vertebrate placodes: insights from developmental studies and from comparisons with other deuterostomes. J Exp Zool B Mol Dev Evol. 2005;304(4):347-99.

95. Dickinson A, Sive H. Positioning the extreme anterior in Xenopus: cement gland, primary mouth and anterior pituitary. Semin Cell Dev Biol. 2007;18(4):525-33.

96. Wardle FC, Sive HL. What's your position? the Xenopus cement gland as a paradigm of regional specification. BioEssays. 2003;25(7):717-26.

97. Yoshida K, Ueno M, Niwano T, Saiga H. Transcription regulatory mechanism of Pitx in the papilla-forming region in the ascidian, Halocynthia roretzi, implies conserved involvement of Otx as the upstream gene in the adhesive organ development of chordates. Dev Growth Differ. 2012;54(6):649-59.

98. Goodyer CG, Tremblay JJ, Paradis FW, Marcil A, Lanctot C, Gauthier Y, et al. Pitx1 in vivo promoter activity and mechanisms of positive autoregulation. Neuroendocrinol. 2003;78(3):129-37.

99. Christiaen L, Bourrat F, Joly JS. A modular cis-regulatory system controls isoform-specific pitx expression in ascidian stomodaeum. Dev Biol. 2005;277(2):557-66.

100. Duboc V, Rottinger E, Besnardeau L, Lepage T. Nodal and BMP2/4 signaling organizes the oral-aboral axis of the sea urchin embryo. Dev Cell. 2004;6(3):397-410 
101. Molina MD, de Croze N, Haillot E, Lepage T. Nodal: master and commander of the dorsal-ventral and left-right axes in the sea urchin embryo. Curr Opin Gen Dev. 2013;23(4):445-53.

102. Saudemont A, Haillot E, Mekpoh F, Bessodes N, Quirin M, Lapraz F, et al. Ancestral regulatory circuits governing ectoderm patterning downstream of Nodal and BMP2/4 revealed by gene regulatory network analysis in an echinoderm. PLoS Genet. 2010;6(12):e1001259.

103. Hibino T, Nishino A, Amemiya S. Phylogenetic correspondence of the body axes in bilaterians is revealed by the right-sided expression of Pitx genes in echinoderm larvae. Dev Growth Differ. 2006;48(9):587-95.

104. Lowe CJ, Terasaki M, Wu M, Freeman Jr RM, Runft L, Kwan K, et al. Dorsoventral patterning in hemichordates: insights into early chordate evolution. PLoS Biol. 2006;4(9):e291.

105. Goodrich ES. "Proboscis pores" in craniate vertebrates, a suggestion concerning the premandibular somites and hypophysis. Q J Micr Sci. 1917;62:539-53.

106. Schlosser G. How old genes make a new head: redeployment of Six and Eya genes during the evolution of vertebrate cranial placodes. Int Comp Biol. 2007;47(3):343-59.

107. Kozmik Z, Holland ND, Kreslova J, Oliveri D, Schubert M, Jonasova K, et al. Pax-Six-Eya-Dach network during amphioxus development: conservation in vitro but context specificity in vivo. Dev Biol. 2007;306(1):143-59.

108. Butts T, Holland PW, Ferrier DE. Ancient homeobox gene loss and the evolution of chordate brain and pharynx development: deductions from amphioxus gene expression. Proc Biol Sci. 2010;277(1699):3381-9.

109. van Wijhe JW. Ueber Amphioxus. Anat Anz. 1893;8:152-72.

110. van Wijhe JW. Die Homologisierung des Mundes des Amphioxus und primitive Leibesgliederung der Wirbelthiere. Petrus Camper. 1906;4:61-102.

111. van Wijhe JW. On the anatomy of the larva of Amphioxus lanceolatus and the explanation of its asymmetry. Proc Kon Akad Wetensch Amsterdam. 1919;21:1013-23.

112. MacBride EW. The formation of the layers in Amphioxus and its bearing on the interpretation of the early ontogenetic processes in other vertebrates. Q J Micr Sci. 1909;54:279-345.

113. Willey A. Amphioxus and the ancestry of the vertebrates. New York: MacMillan and Co.; 1894

114. Veeman MT, Newman-Smith E, El-Nachef D, Smith WC. The ascidian mouth opening is derived from the anterior neuropore: reassessing the mouth/ neural tube relationship in chordate evolution. Dev Biol. 2010;344(1):138-49.

115. Holland ND, Holland LZ. Stage- and tissue-specific patterns of cell division in embryonic and larval tissues of amphioxus during normal development. Evol Dev. 2006;8(2):142-9.

116. Kioussi C, Briata P, Baek SH, Rose DW, Hamblet NS, Herman T, et al. Identification of a Wnt/Dvl/beta-Catenin Pitx2 pathway mediating cell-typespecific proliferation during development. Cell. 2002;111(5):673-85.

117. Suh H, Gage PJ, Drouin J, Camper SA. Pitx2 is required at multiple stages of pituitary organogenesis: pituitary primordium formation and cell specification. Development. 2002;129(2):329-37.

118. Tremblay JJ, Lanctot C, Drouin J. The pan-pituitary activator of transcription, Ptx1 (pituitary homeobox 1), acts in synergy with SF-1 and Pit1 and is an upstream regulator of the Lim-homeodomain gene Lim3/Lhx3. Mol Endocrinol. 1998;12(3):428-41.

119. Candiani S, Holland ND, Oliveri D, Parodi M, Pestarino M. Expression of the amphioxus Pit-1 gene (AmphiPOU1F1/Pit-1) exclusively in the developing preoral organ, a putative homolog of the vertebrate adenohypophysis. Brain Res Bull. 2008;75(2-4):324-30.

120. Monaghan AP, Kioschis P, Wu W, Zuniga A, Bock D, Poustka A, et al. Dickkopf genes are co-ordinately expressed in mesodermal lineages. Mech Dev. 1999;87(1-2):45-56

121. Schweickert A, Steinbeisser H, Blum M. Differential gene expression of Xenopus Pitx1, Pitx2b and Pitx2c during cement gland, stomodeum and pituitary development. Mech Dev. 2001;107(1-2):191-4.

122. Dickinson AJ, Sive HL. The Wnt antagonists Frzb-1 and Crescent locally regulate basement membrane dissolution in the developing primary mouth Development. 2009;136(7):1071-81.

123. Gage PJ, Qian M, Wu D, Rosenberg KI. The canonical Wnt signaling antagonist DKK2 is an essential effector of PITX2 function during normal eye development. Dev Biol. 2008;317(1):310-24.

124. Minguillon C, Garcia-Fernandez J. The single amphioxus Mox gene: insights into the functional evolution of Mox genes, somites, and the asymmetry of amphioxus somitogenesis. Dev Biol. 2002;246(2):455-65.
125. Yasui K, Tabata S, Ueki T, Uemura M, Zhang SC. Early development of the peripheral nervous system in a lancelet species. J Comp Neurol. 1998;393(4):415-25.

126. Kawakami Y, Raya A, Raya RM, Rodriguez-Esteban C, Izpisua Belmonte JC. Retinoic acid signalling links left-right asymmetric patterning and bilaterally symmetric somitogenesis in the zebrafish embryo. Nature. 2005:435 (7039):165-71.

127. Vermot J, Gallego Llamas J, Fraulob V, Niederreither K, Chambon P, Dolle P. Retinoic acid controls the bilateral symmetry of somite formation in the mouse embryo. Science. 2005;308(5721):563-6.

128. Vermot J, Pourquie $\mathrm{O}$. Retinoic acid coordinates somitogenesis and left-right patterning in vertebrate embryos. Nature. 2005;435(7039):215-20.

129. Yoshiba S, Hamada H. Roles of cilia, fluid flow, and Ca2+ signaling in breaking of left-right symmetry. Trends Genet. 2014;30(1):10-7.

doi:10.1186/2041-9139-6-5

Cite this article as: Soukup et al:: The Nodal signaling pathway controls left-right asymmetric development in amphioxus. EvoDevo 2015 6:5.

\section{Submit your next manuscript to BioMed Central and take full advantage of:}

- Convenient online submission

- Thorough peer review

- No space constraints or color figure charges

- Immediate publication on acceptance

- Inclusion in PubMed, CAS, Scopus and Google Scholar

- Research which is freely available for redistribution

Submit your manuscript at www.biomedcentral.com/submit
C Biomed Central 